\title{
ON THE DEGREES AND RATIONALITY OF CERTAIN CHARACTERS OF FINITE CHEVALLEY GROUPS
}

\author{
BY \\ C. T. BENSON( $\left.{ }^{1}\right)$ AND C. W. CURTIS( $\left.{ }^{2}\right)$
}

\begin{abstract}
Let $\mathscr{S}$ be a system of finite groups with $(B, N)$-pairs, with Coxeter system $(W, R)$ and set of characteristic powers $\{q\}$ (see [4]). Let $A$ be the generic algebra of the system, over the polynomial ring $\mathfrak{D}=Q[u]$. Let $K$ be $Q(u), K$ an algebraic closure of $K$, and $\mathfrak{D}^{*}$ the integral closure of $\mathfrak{v}$ in $\bar{K}$. For the specialization $f: u \rightarrow q$ mapping $\mathfrak{D} \rightarrow Q$, let $f^{*}: \mathfrak{D}^{*} \rightarrow \bar{Q}$ be a fixed extension of $f$. For each irreducible character $\chi$ of the algebra $A^{R}$, there exists an irreducible character $\zeta_{x, f^{*}}$ of the group $G(q)$ in the system corresponding to $q$, such that $\left(\zeta_{x, f^{*}}, 1_{B(q)}^{G(q)}\right)>0$, and $\chi \rightarrow \zeta_{x, f^{*}}$ is a bijective correspondence between the irreducible characters of $A^{R}$ and the irreducible constituents of $1_{B(q)}^{G(q)}$. Assume almost all primes occur among the characteristic powers $\{q\}$. The first main result is that, for each $\chi$, there exists a polynomial $d_{x}(t) \in Q[t]$ such that, for each specialization $f: u \rightarrow q$, the degree $\zeta_{x, f^{*}}(1)$ is given by $d_{x}(q)$. The second result is that, with two possible exceptions in type $E_{7}$, the characters $\zeta_{x, f^{*}}$ are afforded by rational representations of $G(q)$.
\end{abstract}

1. Introduction. The results in this paper are concerned with the irreducible complex characters $\chi$ of a finite Chevalley group or a twisted type $G$, with the property that $\chi$ appears with positive multiplicity in the induced permutation representation $1_{B}^{G}$ from a Borel subgroup $B$ of the group $G$. It is proved (with certain exceptions) that these characters are afforded by rational representations of $G$, and that their degrees are polynomials in $q$ with rational coefficients, where $q$ is the order of the finite field associated with $G$.

These results were proved by Steinberg [17] for the general linear groups over a finite field, and had been verified for some other special cases. Some of the methods in this paper were introduced by Curtis, Iwahori and Kilmoyer [4], and explicit formulas for the degrees of certain characters in $1_{B}^{G}$ for a general Chevalley group or twisted type were derived in $[4, \S \S 9,10]$.

From the point of view of Harish-Chandra's approach to characters of finite Chevalley groups using cusp-forms, the results in this paper deal with a special case of problem (b), p. C-19 of Springer [16], that is, splitting up an induced representation $\Omega(c, \omega)=1_{B}^{G}$, where $c$ is the equivalence class of the cuspidal pair $(B, H)$ where

Received by the editors December 14, 1970.

AMS 1970 subject classifications. Primary 20C30; Secondary 20G05.

Key words and phrases. Finite group, $(B, N)$-pair, Coxeter group, generic algebra, irreducible character, generic degree.

(1) The work of the first author was supported in part by a Summer Research Grant from the University of Oregon.

$\left({ }^{2}\right)$ The work of the second author was supported in part by NSF Grants GP-20308 and GP-9535. 
$H$ is a maximal torus in the Borel subgroup $B$, and $\omega$ is the trivial class in the discrete series of the torus $H$.

Here is a sketch of the contents of the paper. $\$ 2$ contains precise statements of the main results, together with most of the definitions and notations used later in the paper. $\S 3$ contains a proof of the result about the degrees of the characters, which is independent of the classification of the Coxeter systems of the groups. $\$ 4$ contains some general results about the generic rings of Coxeter systems, which, together with some results in $\S 2$, achieve a reduction of the proofs of the main theorems to verifying certain facts about finite Coxeter groups. These facts seem to be of independent interest, and are proved for the various types of Coxeter groups using a case-by-case analysis, in the subsequent sections.

It is a pleasure to acknowledge the help we received from Professors J. S. Frame and R. Carter, who furnished us with preprints or proof sheets of some of their forthcoming articles.

2. Statements of results. In general, the notations in [5] will be used. In order to discuss the results from a uniform point of view, we axiomatize the situation as follows (see [4]).

(2.1) Definitions. Let $(W, R)$ be a finite Coxeter system, with finite Coxeter group $W$ and set of distinguished generators $R$. For $w \in W$ we denote by $l(w)$ the length of $w$, i.e. the minimal number of factors among all possible expressions of $w$ as a word in the distinguished generators. A system $\mathscr{S}$ of $(B, N)$-pairs of type $(W, R)$ consists of the Coxeter system $(W, R)$, an infinite set $\mathscr{C} \mathscr{P}$ of prime powers $\{q\}$, called characteristic powers, a set of positive integers $\left\{c_{r}\right\}_{r \in R}$, and for each characteristic power $q$, a finite group $G=G(q)$ with a $(B, N)$-pair, $(B(q), N(q))$, such that $(W, R)$ is the Coxeter system of $G(q)$, and:

(i) $c_{r}=c_{s}$, for $r, s \in R$, if $r$ and $s$ are conjugate in $W$;

(ii) for each group $G=G(q) \in \mathscr{S}$, the index parameters

$$
\text { ind } r=\left[B(q): B(q) \cap B(q)^{r}\right]
$$

for $r \in R$, are given by ind $r=q^{c_{r}}$;

(iii) if $(W, R)$ is of type $G_{2}$, we assume that $c_{r}+c_{s}$ is even, for $r$ and $s$ in $R$.

To such a system corresponds a generic ring $A=A(u)$, which is an algebra with a free basis $\left\{a_{w}\right\}_{w \in W}$, over the polynomial ring $\mathrm{o}=Q[u]$, satisfying, for $w \in W, r \in R$,

$$
\begin{array}{ll}
a_{w} a_{r}=a_{w r}, & l(w r)>l(w), \\
a_{w} a_{r}=u^{c} a_{w r}+\left(u^{c_{r}}-1\right) a_{w}, & l(w r)<l(w) .
\end{array}
$$

For each homomorphism $f: \mathfrak{o} \rightarrow Q$, we can define the specialized algebra over $Q$,

$$
A_{f}=Q \otimes_{0} A,
$$

which has as a $Q$-basis the elements $\left\{a_{w f}\right\}$ given by $a_{w f}=1 \otimes a_{w}, w \in W$. The map $f: \sum u_{w} a_{w} \rightarrow \sum f\left(u_{w}\right) a_{w f}, u_{w} \in \mathfrak{o}$, can be viewed as a homomorphism of algebras over $\mathfrak{v}$, if we view $A_{f}$ as an $\mathfrak{o}$-algebra, with $r a$ defined to be $f(r) a, r \in \mathfrak{v}, a \in A_{f}$. 
Let $f$ be the specialization $u \rightarrow q$ for a characteristic power $q$. Then

$$
A(q)=A_{f} \cong H_{Q}(G, B),
$$

for $G=G(q) \in \mathscr{S}$, where $H_{Q}(G, B)$ denotes the Hecke algebra (or centralizer ring) $e Q G e$, for $e=|B|^{-1} \sum_{x \in B} x$, and is isomorphic to $\operatorname{Hom}_{Q G}\left(1_{B}^{G}, 1_{B}^{G}\right)$.

For the specialization $u \rightarrow 1$, we have $A(1) \cong Q W$, the group algebra of $W$ over $Q$.

Let $K=Q(u), \bar{K}, \bar{Q}$ algebraic closures of $K$ and $Q$ respectively, and o* the integral closure of $\mathrm{o}$ in $\bar{K}$.

The first result to be stated is proved in [4, Proposition (7.1)].

(2.2) Proposition. Let $\chi$ be an irreducible character of the generic algebra $A^{R}$. Then $\chi\left(a_{w}\right) \in \mathfrak{o}^{*}$, for all $w \in W$. Let $f: u \rightarrow q$ or $u \rightarrow 1$ define a specialization belonging to a characteristic power $q$ or 1 . Let $f^{*}: \mathfrak{o}^{*} \rightarrow \bar{Q}$ be an extension of $f$. Then the linear map $\chi_{f}: A_{f}^{\bar{Q}} \rightarrow \bar{Q}$ defined by

$$
\chi_{f}\left(a_{w f}\right)=f^{*}\left(\chi\left(a_{w}\right)\right)
$$

is an irreducible character of $A_{f}^{\bar{Q}}$. For a fixed extension $f^{*}$ of $f$, the map $\chi \rightarrow \chi_{f}$. is a bijection between the irreducible characters of $A^{\bar{K}}$ and those of $A_{f}^{\bar{Q}}$.

From [3] we have

(2.3) Proposition. With the notations as in (2.2), each specialized character $\chi_{f}$., viewed as a character of $H_{\bar{Q}}(G, B)$, is the restriction to $H_{\bar{Q}}(G, B)$ of a unique absolutely irreducible character $\zeta_{x, f}$ of $G(q)$ (or $W$ ), such that $\zeta_{x, f^{*}} \in 1_{B}^{G(q)}$. Every irreducible constituent of $1_{B}^{G}$ is obtained in this way. The degree of $\zeta_{x, f^{*}}$ is given by

$$
\operatorname{deg} \zeta_{x, f^{*}}=[G: B] \operatorname{deg} \chi\left\{\sum_{w \in W}(\text { ind } w)^{-1} \chi_{f} \cdot\left(\hat{a}_{w f}\right) \chi_{f} \cdot\left(a_{w f}\right)\right\}^{-1}
$$

where $\hat{a}_{w f}$ is the basis element of $A_{f}$ corresponding to $w^{-1}$ and

$$
\text { ind } w=\left[B: B \cap B^{w}\right], \quad w \in W .
$$

For the specialization $f_{0}: u \rightarrow 1$, the formula becomes $\operatorname{deg} \zeta_{x_{.} f_{0}}=\operatorname{deg} \chi$.

(2.4) Definition. With the notation as above let

$$
d_{x}=\left[\sum_{w \in W} v\left(a_{w}\right)\right] \operatorname{deg} \chi\left\{\sum_{w \in W} \nu\left(a_{w}\right)^{-1} \chi\left(\hat{a}_{w}\right) \chi\left(a_{w}\right)\right\}^{-1}
$$

where $\nu: A \rightarrow \mathfrak{D}$ is the homomorphism such that $\nu\left(a_{r}\right)=u^{c_{r}}, r \in R . d_{x}$ is called the generic degree associated with $\chi$.

(2.5) Proposition. For each $\chi$ and $f^{*}$, as above, $d_{x}$ belongs to the specialization ring of $f^{*}$, and $f^{*}\left(d_{\chi}\right)=\operatorname{deg}\left(\zeta_{x, f^{*}}\right)$.

Proof. From [4, Lemmas (5.2) and (5.9)], we have $f\left(\sum_{w \in W} \nu\left(a_{w}\right)\right)=[G: B]$, and $f\left(\nu\left(a_{w}\right)\right)=$ ind $w$. The rest of the proof is clear, taking into account the formula for $\operatorname{deg} \zeta_{x, f}$ given in Proposition (2.3), and Proposition (2.2). 
(2.6) THEOREM. Suppose the set of characteristic powers of $\mathscr{S}$ contains almost all primes. Then for each irreducible character $\chi$ of $A^{R}$, the generic degree $d_{x} \in Q[u]$.

Note that this theorem does not apply to twisted $G_{2}, B_{2}$ or $F_{4}$, but the conclusion is true for twisted $G_{2}$ or $B_{2}$ since in these cases, the representation of $G$ on the cosets of $B$ is doubly transitive. In the case of twisted $F_{4}$, the generic degrees do not belong to $\mathrm{o}$ (see $[4, \S 9])$.

Definition. A character $\chi$ of $A^{R}$ is said to be rational in case $\chi\left(a_{w}\right) \in \mathfrak{v}$ for all $w \in W$.

Note that if $\chi$ is rational, then the specialized characters $\chi_{f}$. defined in Proposition (2.2) have the property that $\chi_{f}$. is independent of the extension $f^{*}$ of $f$, and $\chi_{f} \cdot\left(a_{w f}\right)$ $\in Q$ for all $w \in W$.

(2.7) THEOREM. Suppose $\chi$ is an irreducible character of $A^{R}$ and $J \subset R$. Set $e_{J}=\sum_{w \in W_{J}} a_{w}, E_{J}=\nu\left(e_{J}\right)$ and let $m_{J}(\chi)$ be the nonnegative integer such that $\chi\left(e_{J}\right)$ $=m_{J}(\chi) E_{J}$. Suppose for each irreducible character $\chi^{\prime} \neq \chi$, there exists $a J \subset R$ such that $m_{J}(\chi) \neq m_{J}\left(\chi^{\prime}\right)$. Then $\chi$ is a rational character of $A_{R}$, i.e. $\chi\left(a_{w}\right) \in \mathfrak{0}, w \in W$.

Proof. For each specialization $f: u \rightarrow q$ or $1, m_{J}(\chi)=\left(1_{G_{J}}^{G}, \zeta_{\chi, f^{*}}\right)$ where $G_{J}$ is the parabolic subgroup $B W_{J} B$ (see $[4, \S 7]$ ). Suppose $\chi$ is not rational; then $\chi \neq \chi^{\sigma}$ for some field automorphism $\sigma$ of $\bar{K}$ over $K$. But $m_{J}(\chi)=m_{J}\left(\chi^{\sigma}\right)$ for all subsets $J \subset R$, contradicting the hypothesis. This completes the proof.

Using Theorem (2.7), the following theorem can be proved.

(2.8) Theorem. Suppose $(W, R)$ is of type $A_{n}(n \geqq 1), B_{n}\left(\right.$ or $\left.C_{n}\right)(n \geqq 2), D_{n}(n \geqq 4)$, $G_{2}, F_{4}, E_{6}, E_{7}, E_{8}$. Then every irreducible character of the generic algebra $A^{R}$ is rational, with the possible exception of the two irreducible characters of degree 512, in case $(W, R)$ is of type $E_{7}$.

In case $W$ is of type $E_{7}$, it is the direct product of a cyclic group $C$ of order 2 and the Chevalley group $B_{3}(2)$. If $1_{C}$ and $\varepsilon_{C}$ are the two irreducible characters of $C$ and $\zeta$ is the Steinberg character of $B_{3}(2)$, the characters $1_{C} \otimes \zeta$ and $\varepsilon_{C} \otimes \zeta$ of $W$ occur with the same multiplicity in $1_{P}^{W}$ for every parabolic subgroup $P$ of $W$. The two characters of $A^{R}$ corresponding to $1_{C} \otimes \zeta$ and $\varepsilon_{C} \otimes \zeta$ fail to satisfy the hypothesis of Theorem (2.7) and account for the exception in Theorem (2.8).

We remark that Theorem (2.8), combined with the formula for generic degrees (2.4), gives an independent proof of Theorem (2.6) (on the rationality of generic degrees) for generic rings of the type listed in Theorem (2.8), with the possible exception of the two characters of degree 512 in the case of $E_{7}$.

Before stating the next theorem, some new concepts have to be introduced. An irreducible character $\chi$ of a finite dimensional algebra $A$ over a field $K$ is said to be afforded by a primitive idempotent $e \in A$ in case $\chi$ is the character of the irreducible representation of $A$ on the minimal left ideal $A e$ in $A$. Now suppose $K$ is the quotient field of a principal ideal domain $R$, and that $A$ has a $K$-basis $\left\{a_{1}, \ldots, a_{n}\right\}$ for which 
the structure constants all belong to $R$. For each $a \in A$, we let $\delta(a)$ denote a generator of the ideal in $R$ consisting of all elements $x \in R$ such that $x a \in \sum R a_{i} ; \delta(a)$ will be called the denominator of $a$.

(2.9) THEOREM. Let $\mathscr{S}$ be a system of groups with $(B, N)$-pairs of any one of the types $A_{n}(n \geqq 1), B_{n}\left(\right.$ or $\left.C_{n}\right)(n \geqq 2), D_{n}(n \geqq 4), G_{2}, F_{4}, E_{6}, E_{7}$, or $E_{8}$. Then every irreducible character $\chi$ of the generic algebra $A^{R}$ is afforded by a primitive idempotent $a_{x} \in A^{K}$, with the possible exception of the two characters of degree 512 in case $\mathscr{S}$ is of type $E_{7}$. Thus, except for the case of $E_{7}, K$ is a splitting field for $A^{K}$. Let $a_{x}$ be any one of these rational idempotents, and let $f$ be a specialization associated with a characteristic power $q$ or 1 . Then $f\left(\delta\left(a_{x}\right)\right) \neq 0$, and $f\left(\delta\left(a_{x}\right)\right)^{-1} f\left[\delta\left(a_{x}\right) a_{x}\right]=a_{x f}$ is a primitive idempotent in the group algebra $Q G(q)$, affording the irreducible complex character $\zeta_{x, f}$ of $G$. Thus every irreducible character $\zeta \in 1_{B}^{G}$, for $G \in \mathscr{S}$, is afforded by a rational representation of $G$, with the possible exception of two characters of $G$ associated with the characters of degree 512 of the generic algebra, in case $\mathscr{S}$ is of type $E_{7}$.

The methods to be given below provide explicit formulas for the idempotents $a_{x}$, in terms of the characters of $A^{R}$ and its subalgebras $A_{J}^{R}=\left\langle a_{r} ; r \in J\right\rangle^{R}, J \subset R$.

As a corollary to the proof of Theorem (2.9), the following results can be stated.

(2.10) Corollary. Let $(W, R)$ be a Coxeter system of type $A_{n}(n \geqq 1), B_{n}(n \geqq 2)$, $D_{n}(n \geqq 4)$, or $G_{2}, F_{4}, E_{6}, E_{7}, E_{8}$. Let $\chi$ be an irreducible complex character of $W$. There exist subsets $R=J_{1} \supset J_{2} \supset \cdots \supset J_{s}$ of $R$, for $s=2$ or 3 , and irreducible characters $\chi_{1}=\chi, \chi_{2}, \ldots, \chi_{s}$ of $W_{J_{1}}, \ldots, W_{J_{s}}$ respectively, such that $\left(\chi_{i}, \chi_{i+1}^{W_{J_{i}}}\right)=1$, for $1 \leqq i \leqq s-1$, with $\chi_{s}$ a linear character of $W_{J_{s}}$. Let $e_{\chi}$ be the idempotent $e\left(\chi_{1}\right) e\left(\chi_{2}\right) \cdots e\left(\chi_{s}\right)$, where

$$
e\left(\chi_{i}\right)=\chi_{i}(1)\left|W_{J_{i}}\right|^{-1} \sum_{x \in W_{J_{i}}} \chi_{i}\left(x^{-1}\right) x .
$$

Then $e_{\chi}$ is primitive, belongs to $Q W$, and affords $\chi$. Thus each irreducible character of $W$ is afforded by a rational representation.

The statements about the existence of the characters $\left\{\chi_{i}\right\}$ and their multiplicities are proved in subsequent sections. The fact that $e_{\chi}$ is a primitive idempotent affording $\chi$ follows from a result of Janusz [11]. The third statement, that $e_{x} \in Q W$, is a consequence of the known result that all the characters of $W$ are rational (without exception).

We remark that the proof of Theorem (2.8) given below for $(W, R)$ of type $A_{n}$, $B_{n}$ and $D_{n}$ gives a new proof of the rationality of the characters of Coxeter groups of these types. The last statement of Corollary (2.10), on the rationality of the representations of the Coxeter groups of the types listed, is not a new result. The last open problems on the subject were settled by Benard [1] for types $E_{6}, E_{7}$ and $E_{8}$, by an analysis of the elementary subgroups of these Coxeter groups and using some results about Schur indices. The result was already known for the other types (see [1] for references). 
(2.11) COROLlary. Let the hypothesis of Theorem (2.9) be satisfied. Then $H_{Q}(G(q), B(q)) \cong Q W$, except possibly for $W$ of type $E_{7}$.

To prove this corollary, one first observes that by the Deformation Theorem (see Steinberg [18, p. 249]), both $H_{Q}(G(q), B(q))$ and $Q W$ have the same numerical invariants. It then follows immediately from Theorem (2.9) and Corollary (2.10) that, with the possible exception of $E_{7}$, the field of rational numbers $Q$ is a splitting field for $H_{Q}(G(q), B(q))$ and $Q W$, and the corollary follows.

We conclude this section by calling attention to two of the combinatorial methods and results used in later sections to discuss the various types of Coxeter groups. The first is the systematic use of Clifford's theorem in the form given by Gallagher [10, Theorem 2] on characters of semidirect products, for computation of characters of $B_{n}$ in $\S \S 6$ and 7 . The second is the following result about characters of the symmetric group (Theorem (6.7) below).

(2.12) Proposition. Suppose $\omega$ and $\omega^{\prime}$ are characters of the symmetric group $S_{n}$, $n \geqq 3$, with the property that $\omega(p) / \omega(1)=\omega^{\prime}(p) / \omega^{\prime}(1)$, for all cycles $p$ of lengths $2,3, \ldots, n-1$. Then $\omega=\omega^{\prime}$.

3. Rationality of generic degrees. This section contains a proof of Theorem (2.6). Let $\chi$ be an irreducible character of $A^{R}$, and let

$$
c_{\chi}=\sum_{w \in W} \nu\left(a_{w}\right)^{-1} \chi\left(\hat{a}_{w}\right) \chi\left(a_{w}\right)
$$

We assert first that it is sufficient to prove that $c_{x} \in K=Q(u)$. For if that is the case, then $d_{x} \in K$, and is a rational function in $Q(u)$ which becomes an integer upon substitution of infinitely many integer values of $u$. An elementary argument (which is omitted) then shows that $d_{x} \in Q[u]$.

From Proposition (2.2), we have $\chi\left(a_{w}\right) \in \mathfrak{o}^{*}, w \in W$, where $\mathfrak{o}^{*}$ is the integral closure of $\mathrm{o}$ in $\bar{K}$. Therefore, letting $c_{x}^{\prime}=\left[\mathrm{Icm}_{w \in W} \nu\left(a_{w}^{\prime}\right)\right] c_{x}$, we have $c_{x}^{\prime} \in \mathfrak{o}^{*}$, and it is sufficient to prove that $c_{x}^{\prime} \in K$. Since $c_{x}^{\prime} \in \mathfrak{v}^{*}$, we have $\operatorname{Irr}\left(c_{x}^{\prime}, K, t\right) \in \mathfrak{o}[t]$. Letting

$$
F(u, t)=\operatorname{Irr}\left(c_{x}^{\prime}, K, t\right)=t^{m}+\varphi_{1}(u) t^{m-1}+\cdots+\varphi_{m}(u), \quad \varphi_{i}(u) \in \mathfrak{v},
$$

we see that $F(u, t)$ is an irreducible polynomial in $Q[u, t]$.

Now let $f: u \rightarrow q$ be a specialization, for some $q \in \mathscr{C} \mathscr{P}$, and let $f^{*}: \mathfrak{o}^{*} \rightarrow \bar{Q}$ be an extension of $f$. Then $f^{*}\left(c_{x}^{\prime}\right) \in Q$, since $f^{*}\left(d_{x}\right) \in Z$, by Proposition (2.5).

Suppose the theorem is false, and that $c_{x}^{\prime} \notin K$. Then $m=\operatorname{deg} \operatorname{Irr}\left(c_{x}^{\prime}, K, t\right)>1$. Let $f: u \rightarrow q$ be a specialization, for $q \in \mathscr{C P P}$, and $f^{*}$ an extension of $f$ to $\mathfrak{D}^{*}$. Then the rational number $f^{*}\left(c_{x}^{\prime}\right)$ is a zero of the polynomial $F(q, t) \in Q[t]$, where $F(q, t)$ is monic, and of degree $m>1$. On the other hand, the Hilbert Irreducibility Theorem (Lang [13, p. 148]) implies that for infinitely many primes $p$, the specialized polynomial $F(p, t)$ is irreducible in $Q[t]$. Some such $p$ must belong to $\mathscr{C} \mathscr{P}$, by the assumption that $\mathscr{C} \mathscr{P}$ contains almost all primes, and we have arrived at a contradiction. This completes the proof of Theorem (2.6). 
4. Primitive idempotents and multiplicities in generic algebras. In this section, some general results about idempotents in generic algebras will be proved, which will provide the means of establishing Theorem (2.9) for each of the individual types of systems of groups with $(B, N)$-pairs. We begin with some general (and presumably well-known) lemmas. Let $K$ be the quotient field of a principal ideal domain $\mathfrak{v}$. Let $A$ be a finite dimensional algebra over $K$, with a basis $\left\{a_{1}, \ldots, a_{n}\right\}$ whose constants of structure lie in $\mathfrak{v}$. Let $B$ be the subring $\sum \mathfrak{v} a_{i}$ of $A$. As in $\S 2$, for $a \in A$ we let $\delta(a)$ be a generator of the ideal consisting of all $x \in R$ such that $x a \in B$. An element $b \in B$ is said to have unit content if $b=\sum r_{i} a_{i}, r_{i} \in \mathbb{0}$, and $\operatorname{gcd}\left\{r_{i}\right\}=1$ $(1 \leqq i \leqq n)$.

(4.1) Lemma. Let $e$ be an idempotent $(\neq 0)$ in $A$. Then $e^{\prime}=\delta(e) e \in B$, and has unit content.

Proof We have $\left(e^{\prime}\right)^{2}=\delta(e) e^{\prime}$. Let $d$ be the g.c.d. of the coefficients of $e^{\prime}$. Then $(d, \delta(e))=1$, otherwise $[\delta(e) /(d, \delta(e))] e \in B$, contrary to the definition of $\delta(e)$. We have $e^{\prime}=d e^{\prime \prime}$, with $e^{\prime \prime}$ of unit content, and $\left(e^{\prime}\right)^{2}=\delta(e) e^{\prime}=d^{2}\left(e^{\prime \prime}\right)^{2}$, which implies that $\delta(e) e^{\prime \prime}=d\left(e^{\prime \prime}\right)^{2}$. Then $d$ is a unit, since $e^{\prime \prime}$ has unit content.

(4.2) Lemma. Let $b$ be an element of unit content in $B$. Then $K b \cap B=\mathfrak{o} b$.

Proof. Let $s^{-1} r b=b^{\prime} \in B$, where $r, s \in \mathfrak{v}$ and $(r, s)=1$. Then $r b=s b^{\prime}$, and since $b$ has unit content, $s \mid r$. Therefore $s$ is a unit, and $s^{-1} r \in \mathfrak{v}$.

We turn now to a system of groups with $(B, N)$-pairs of type $(W, R)$ in the sense of Definition (2.1), and let $A$ be the generic ring associated with the system. We shall apply Lemmas (4.1) and (4.2) to the pair $\left(A^{K}, A\right)$ with $A$ playing the role of $B$, and $K=Q(u)$, the quotient field of $\mathfrak{v}=Q[u]$.

(4.3) Lemma. Let $J \subset R$. Then

(i) $A_{J}=\left\langle a_{r}, r \in J\right\rangle$ is a generic ring of a system of type $\left(W_{J}, J\right)$, with the same set of characteristic powers, where for each $q \in \mathscr{C} \mathscr{P}$, the corresponding group with a $(B, N)$ pair is the parabolic subgroup $G_{J}(q)=B(q) W_{J} B(q)$ of $G(q)$.

(ii) For each specialization $f: u \rightarrow q$ or $1, A_{J f}$ is isomorphic to, and will be identified with, $H_{Q}\left(G_{J}(q), B(q)\right)$ or $W_{J}$ respectively.

The proof of this lemma is entirely routine and will be omitted.

(4.4) Proposition. Let $A, A_{J}$ be as above. Let $\chi$ and $\varphi$ be irreducible characters of $A^{R}$ and $A_{J}^{R}$ respectively, and suppose $\varphi$ is afforded by a primitive idempotent e belonging to $A_{J}^{K}$. Let $e^{\prime}=\delta(e) e$. Then

$$
\chi\left(e^{\prime}\right)=m(\chi, \varphi) \delta(e),
$$

where $m(\chi, \varphi)$ is a nonnegative integer. For every specialization $f: u \rightarrow q$ or 1 , let $f^{*}$ denote a fixed extension of $f$ to the integral closure $\mathfrak{v}^{*}$ of $\mathfrak{v}$ in $\bar{K}$. Then from $\$ 2$, there are corresponding irreducible characters $\zeta_{x, f^{*},} \eta_{\varphi, f^{*}}$ of $G(q)$ and $G_{J}(q)$ respectively. For every specialization $f$ such that $f(\delta(e)) \neq 0$, we have

$$
m(\chi, \varphi)=\left(\zeta_{\chi, f^{*},} \eta_{\varphi, f^{*}}^{G(q)}\right)
$$


Proof. For $a \in A$ or $A_{J}$, let $a_{f}$ denote the image in the specialized algebra $A_{f}$ or $A_{J f}$ of $a$, under the homomorphism of o-algebras defined by $f$. We prove first that in case $f(\delta(e)) \neq 0, e_{f}^{\prime} \mid f(\delta(e))$ is a primitive idempotent in $A_{J f}=H_{Q}\left(G_{J}(q), B(q)\right)$ affording the character $\eta_{\varphi, f^{*}}$

We have $e^{\prime} A_{J} e^{\prime}=K e^{\prime} \cap A_{J}=$ ve $e^{\prime}$ by Lemma (4.2). Moreover $\left(e^{\prime}\right)^{2}=\delta(e) e^{\prime}$. Applying the homomorphism $f$ to these equations, and using the fact that $e_{f}^{\prime} \neq 0$ since $e^{\prime}$ has unit content (by Lemma (4.1)), it follows that $e_{f}^{\prime} / f(\delta(e)$ ) is a primitive idempotent in $A_{J f}$.

Finally, since $\varphi(e)=1$, we have $\varphi\left(e^{\prime}\right)=\delta(e)$, and hence

$$
\eta_{\varphi, f^{*}}\left(e_{f}^{\prime}\right)=\varphi_{f \bullet}\left(e_{f}^{\prime}\right)=f(\delta(e)) \neq 0 .
$$

This completes the proof that $e_{f}^{\prime} \mid f(\delta(e))$ is primitive and affords $\eta_{\varphi, j}$.

We next have from $\chi\left(e^{\prime}\right)=m(\chi, \varphi) \delta(e)$ the result that

$$
\zeta_{\chi, f^{*}}\left(e_{f}^{\prime}\right)=\chi_{f} \cdot\left(e_{f}^{\prime}\right)=m(\chi, \varphi) f(\delta(e)) .
$$

From this formula we conclude, in case $f(\delta(e)) \neq 0$, that $m(\chi, \varphi)=\left(\zeta_{\chi, f^{*},}, \eta_{\varphi, f^{*}}^{G(q)}\right)$, and the proposition is proved.

For an irreducible character $\chi$ of $A^{R}$, we shall denote by $e(\chi)$ the central primitive idempotent corresponding to $\chi$.

(4.5) Proposition. Let $A, A_{J}, \chi, \varphi, e$ be as above, and suppose that the irreducible character $\chi$ of $A^{R}$ is rational, in the sense that $\chi\left(a_{w}\right) \in \mathfrak{v}$ for all $w \in W$. Finally suppose the multiplicity $m(\chi, \varphi)=1$. Then $e(\chi) e$ is a primitive idempotent in $A^{K}$ affording $\chi$. Let $f: u \rightarrow q$ or 1 be a specialization such that $f(\delta(e)) \neq 0$. Then $f(\delta(e(\chi) e)) \neq 0$, and, letting $\tilde{e}=\delta(e(\chi) e) e(\chi) e, \tilde{e}_{f} \mid f(\delta(e(\chi) e))$ is a primitive idempotent in $A_{f}=H_{Q}(G(q), B(q))$ affording $\zeta_{x, f} \cdot$

Proof. From Corollary (5.12) of [4], with $J=\varnothing$, we know that $A^{R}$ is a split semisimple symmetric algebra, with dual bases $\left\{a_{w}\right\}$ and $\left\{\nu\left(a_{w}\right)^{-1} \hat{a}_{w}\right\}$ with respect to the bilinear form defined in [4]. From Fossum [6], the block idempotent $e(\chi)$ is given by

$$
e(\chi)=\chi(1) c_{\chi}^{-1} \sum_{w \in W} \nu\left(a_{w}\right)^{-1} \chi\left(\hat{a}_{w}\right) a_{w}
$$

where $c_{\chi}=\sum_{w \in W} \nu\left(a_{w}\right)^{-1} \chi\left(\hat{a}_{w}\right) \chi\left(a_{w}\right) \in K$. These remarks show that $e(\chi) \in A^{K}$ if $\chi$ is rational. Let $m=\operatorname{lcm}_{w \in W}\left\{\nu\left(a_{w}\right)\right\}$. Then $f(m) \neq 0, m c_{\chi} \in \mathfrak{0}$, and $f\left(m c_{\chi}\right) \neq 0$ because of Proposition (2.5), and the formula for the generic degree $d_{x}$. It follows that

$$
m c_{\chi} \cdot \delta(e) e e(\chi) \in A,
$$

and hence $\delta(e e(\chi)) \neq 0$ since $f\left(m c_{\chi} \delta(e)\right) \neq 0$.

Next, $e \in A_{J}^{K}$ affords $\varphi$ and $\chi(e)=1$, by our hypothesis. This means that if $E$ is a primitive idempotent in $A^{R}$ affording $\chi$, then $\operatorname{dim} e A^{R} E=1$, and by [5, (54.16)], the number of composition factors of $A^{R} E$ isomorphic to $A_{J}^{R} e$ is one. 
We can now state the following lemma, which is an immediate consequence of Theorems 6, 7, and 8 of Nakayama [15], and the facts that $A_{J}^{R}$ and $A^{R}$ are semisimple algebras over an algebraically closed field $\bar{K}$.

(4.6) Lemma. Let $A_{J}^{R}, A^{R}$, $e$ and $E$ be as above. Then the number of composition factors of $A^{R} E$ which are isomorphic to $A_{J}^{R} e$ is equal to the number of composition factors of $e A^{R}$ isomorphic to $E A^{R}$.

Applying the lemma, we conclude $e(\chi) e A^{R}$ is a minimal right ideal in $A^{R}$, and hence $A^{R} e(\chi) e$ is a minimal left ideal affording the character $\chi$. Now let $f$ be a specialization such that $f(\delta(e)) \neq 0$. From what has been proved, and from the proof of Proposition (4.4), it follows that $\tilde{e}_{f} / f(\delta(e(\chi) e))$ is a primitive idempotent in $A_{f}$. It remains to check that it affords the character $\zeta_{\chi, f}$. Since $\tilde{e} / \delta(e(\chi) e)$ affords $\chi$, we have $\chi(\tilde{e})=\delta(e(\chi) e)$. Therefore

$$
\chi_{f} \cdot\left(\tilde{e}_{f}\right)=f(\delta(e(\chi) e))
$$

and hence, for a specialization $f$ satisfying our hypotheses,

$$
\zeta_{x, f \cdot}\left(\tilde{e}_{f}\right)=\chi_{f \cdot} \cdot\left(\tilde{e}_{f}\right)=f(\delta(e(\chi) e)) \neq 0 .
$$

This completes the proof of the proposition.

In the applications of these results in subsequent sections, the construction of idempotents via Proposition (4.5) will always begin with some subset $J \subset R$ for which the character $\varphi$ of $A_{J}$ is either $\nu: A_{J} \rightarrow \mathfrak{o}$ or $\varepsilon: A_{J} \rightarrow \mathfrak{p}$, given by $\nu\left(a_{r}\right)=u^{c_{r}}$, $r \in R$, and $\varepsilon\left(a_{r}\right)=-1, r \in R$, respectively. The idempotents $e$ affording these characters are

$$
\left[\sum_{w \in W_{J}} \nu\left(a_{w}\right)\right]^{-1} \sum_{w \in W_{J}} a_{w}
$$

and

$$
\left[\sum_{w \in W_{J}} \nu\left(a_{w}\right)^{-1}\right]^{-1} \sum_{w \in W_{J}} \nu\left(a_{w}\right)^{-1}(-1)^{l(w)} a_{w}
$$

respectively, and thus $f(\delta(e)) \neq 0$ for all specializations $f: u \rightarrow q$ or 1 . This means that the hypothesis about $f$ needed to apply Proposition (4.5) will automatically be satisfied in the applications given in later sections.

The rationality of an irreducible character of a generic algebra $A^{R}$, when it is needed to apply Proposition (4.5), will follow from previously checked cases of Theorem (2.8), and the following result.

(4.7) Proposition. Let $A$ be a generic ring of type $(W, R)$, and suppose that $R=J_{1} \cup \cdots \cup J_{k}$, with $\left\{J_{s}\right\}$ indecomposable, $\left(J_{s}, J_{s^{\prime}}\right)=1$, and $J_{s} \cap J_{s^{\prime}}=\varnothing$ for $s \neq s^{\prime}$. Then $A \cong A_{J_{1}} \otimes \cdots \otimes A_{J_{k}}$. If all the irreducible characters of $A_{J_{s}}, 1 \leqq s \leqq k$, are rational, the same is true of $A^{R}$.

The proof is omitted. 
5. Proof of Theorems (2.8) and (2.9) for $A_{n}$. We first remark that since $m_{J}(\chi)=\left(1_{G}^{G}, \zeta_{\chi, f^{*}}\right)$ (see the proof of Theorem (2.7)) for all specializations $f: u \rightarrow q$ or $u \rightarrow 1$, the proof of Theorem (2.8) is reduced, using Theorem (2.7), to showing that an irreducible character $\omega$ of $W$ is determined by the multiplicities $m_{J}(\omega)=\left(\omega, 1_{W_{J}}^{W}\right)$. Theorem $(2,8)$ for $W$ of type $A_{n}$ thus follows from the following proposition.

(5.1) Proposition. Let $(W, R)$ be of type $A_{n}$ and let $R=\left\{w_{1}, \ldots, w_{n}\right\}$, indexed so that $\left(w_{i} w_{i+1}\right)^{3}=1, i=1, \ldots, n-1 ;\left(w_{i} w_{j}\right)^{2}=1,|j-i|>1$. Then, for irreducible characters $\omega$; $\omega^{\prime}$ of $W, m_{J}(\omega)=m_{J}\left(\omega^{\prime}\right)$ for all $J \subset R$ implies $\omega\left(w_{J}\right)=\omega^{\prime}\left(w_{J}\right)$ where $w_{J}$ is the product in any order of the elements in J. Moreover $\omega=\omega^{\prime}$.

Proof. In this case $W$ is the symmetric group $S_{n+1}$, and the $w_{i}$ are the transpositions $(i, i+1)$. For a given subset $J \subset R$, the possible choices of $w_{J}$ are all conjugate, and every element of $W_{J}$ which is not conjugate to $w_{J}$ is conjugate to a $w_{J^{\prime}}$, for $J^{\prime} \subset J, J^{\prime} \neq J$. The proposition follows by induction on $|J|$, using the fact that

$$
m_{J}(\omega)=\omega\left(e_{J}\right), \quad e_{J}=\left|W_{J}\right|^{-1} \sum_{w \in W_{J}} w .
$$

We now prove Theorem (2.9) for the case $A_{n}$.

(5.2) Definition. The irreducible character $\zeta_{x_{*}}$. is said to be of parabolic type if $\left(\zeta_{x, f^{*},} 1_{G_{j}}^{G}\right)=1$ for some $J \subset R$.

Using Propositions (4.4), (4.5) and (4.7), Theorem (2.9) for the case $W=A_{n}$ follows from the following proposition.

(5.3) Proposition. Every irreducible character $\omega$ of $(W, R)$ of type $A_{n}$ is of parabolic type.

Proof. To $\omega$ we associate a standard Young tableau $D$, [7]. Then the group $R(D)$ of row permutations of $D$ coincides with $W_{J}$ for some $J$. Moreover $1_{W_{J}}^{W}$ is a Frobenius compound character [7], and $\left(1_{W_{J}}^{W}, \omega\right)=1$ [7] (or [11]).

6. Rationality of characters of a generic algebra of type $B_{n}$. If $(W, R)$ is of type $B_{n}$, we may consider $W$ as the group of signed permutations of $n$ letters [2], and $W=N S_{n}$, the semidirect product of an elementary abelian 2-group $N$ by the symmetric group on $n$ letters. Moreover, $N$ has a basis over $Z_{2}$ consisting of the negative 1 -cycles $\{-(1),-(2), \ldots,-(n)\}$ and we may take the set $R$ to be $\left\{w_{1}, \ldots, w_{n}\right\}$, $w_{i}=(i, i+1), i=1, \ldots, n-1$, and $w_{n}=-(n)$. From [2], two elements are conjugate in $W$ if and only if they have the same signed cycle type.

By one of Clifford's theorems in the form given by Gallagher [10, Theorem 2], the irreducible characters of $W$ are constructed as follows. Each irreducible character appears with positive multiplicity in a unique induced character $\psi^{w}$, where $\{\psi\}$ are representatives of the different equivalence classes, under conjugacy by $W$, of linear characters of $N$. For the representatives $\{\psi\}$, we may select characters $\left\{\psi_{k l}\right\}$ for $k+l=n$, such that $\psi_{k l}(-(i))=1,1 \leqq i \leqq k$, and $\psi_{k l}(-(i))=-1, k+1 \leqq i \leqq n$. For 
such a character $\psi_{k l}$, the centralizer $T_{k l}$ in $W$ is the semidirect product $N S$, where $S \subset S_{n}$, and $S \cong S_{k} \times S_{l}$, the direct product of symmetric groups on $\{1, \ldots, k\}$ and $\{k+1, \ldots, n\}$. We shall write $S=S_{k} \times S_{l}$. Every irreducible constituent of $\psi_{k l}^{W}$ has the form

$$
\eta=\left(\tilde{\psi}_{k l} \omega\right)^{w}=\left(\tilde{\psi}_{k l} \omega_{k} \omega_{l}\right)^{w}
$$

where $\tilde{\psi}_{k l}$ is the extension of $\psi_{k l}$ to $T_{k l}$ such that $\psi_{k l} \mid S=1$, and $\omega$ is an irreducible character of $S=S_{k} \times S_{l}$, and can be expressed in the form $\omega_{k} \omega_{l}$, where $\omega_{k}$ and $\omega_{l}$ are uniquely determined irreducible characters of the symmetric groups $S_{k}$ and $S_{l}$ respectively. We ask the reader to live with the possibility that $k=l$ does not imply that the characters $\omega_{k}$ and $\omega_{l}$ are the same.

The subgroup $T_{k l}$ is the direct product of the groups of signed permutations on $\{1, \ldots, k\}$ and $\{k+1, \ldots, n\}$ respectively, and we shall use the notation $T_{k l}=B_{k} \times B_{l}$. The meaning of the notations $S_{k} \times B_{l}, S_{k} \times S_{l}$, etc., for subgroups of $T_{k l}$ will be clear.

For complementary subsets $K$ and $L$ of $\{1, \ldots, n\}$, define $\psi_{K L}: N \rightarrow\{ \pm 1\}$ to be the character such that $\psi_{K L}(-(i))=1, i \in K$, and $\psi_{K L}(-(i))=-1, i \in L$. Then $\psi_{K L} \cong \psi_{|K|,|L|}$, and $\psi_{K L}^{W}=\psi_{|K|,|L|}^{W}$.

As in $\S 5$, to prove Theorem (2.8) for the case $W=B_{n}$, it is sufficient to show that the irreducible characters $\eta$ of $W$ are determined by their multiplicities $\left(1_{W}^{W}, \eta\right)$. Thus assume, for Lemmas (6.1) and (6.4), that $\eta=\left(\tilde{\psi}_{k l} \omega_{k} \omega_{l}\right)^{W}$ and $\eta^{\prime}=\left(\tilde{\psi}_{k^{\prime} l^{\prime}} \omega_{k^{\prime}}^{\prime} \omega_{l^{\prime}}^{\prime}\right)^{W}$ and that $\left(1_{P}^{W}, \eta\right)=\left(1_{P}^{W}, \eta^{\prime}\right)$ for all parabolic subgroups $P$ of $W$.

(6.1) LemMA. $k=k^{\prime}, l=l^{\prime}$ and $\omega_{k}(1) \omega_{l}(1)=\omega_{k}^{\prime}(1) \omega_{l}^{\prime}(1)$.

Proof. If $P=\{1\}$, we have

$$
\eta(1)=\left[W: T_{k l}\right] \omega_{k}(1) \omega_{l}(1)=\eta^{\prime}(1)=\left[W: T_{k^{\prime} l^{\prime}}\right] \omega_{k^{\prime}}^{\prime}(1) \omega_{l^{\prime}}^{\prime}(1) .
$$

Letting $P=\left\{1, w_{n}\right\}$ we have $\eta\left(w_{n}\right)=\eta^{\prime}\left(w_{n}\right)$. But

$$
\begin{aligned}
\eta\left(w_{n}\right) & =\frac{\omega_{k}(1) \omega_{l}(1)}{\left|T_{k l}\right|} \sum_{x \in W} \tilde{\psi}_{k l}\left(x^{-1} w_{n} x\right) \\
& =\left(\left|C_{W}\left(w_{n}\right)\right| /\left|T_{k l}\right|\right) \omega_{k}(1) \omega_{l}(1)(k-l)
\end{aligned}
$$

where $C_{W}\left(w_{n}\right)$ is the centralizer in $W$ of $w_{n}$. Setting $\eta\left(w_{n}\right)=\eta^{\prime}\left(w_{n}\right)$ and using (6.2) yields $k-l=k^{\prime}-l^{\prime}$ which implies $k=k^{\prime}, l=l^{\prime}$ since $l=n-k, l^{\prime}=n-k^{\prime}$. Then $\omega_{k}(1) \omega_{l}(1)=\omega_{k}^{\prime}(1) \omega_{l}^{\prime}(1)$ from (6.2).

We write $p$ for a cycle of length $p$ in either $S_{k}$ or $S_{l}$ and set $\bar{\omega}_{k}(p)=\omega_{k}(p) / \omega_{l}(1)$, $\bar{\omega}_{l}(p)=\omega_{l}(p) / \omega_{l}(1)$, etc.

(6.4) LeMMA. $\bar{\omega}_{k}(p)=\bar{\omega}_{k}(p)$ and $\bar{\omega}_{l}(p)=\bar{\omega}_{l}^{\prime}(p)$ if the cycle length of $p$ is less than $n$.

Proof. Taking $P=\left\langle w_{1}, \ldots, w_{p-1}, w_{n}\right\rangle$ we obtain, by an argument similar to Proposition (5.1), that for $p=(1, \ldots, p)=w_{1} \cdots w_{p-1}, p \leqq n-1, \eta(p)=\eta^{\prime}(p)$ and $\eta\left(p w_{n}\right)=\eta^{\prime}\left(p w_{n}\right)$. Computing $\eta(p)$ and $\eta\left(p w_{n}\right)$ we obtain

$$
\eta(p)=2^{p-1}(p-1) ! \frac{\left|C_{w}(p)\right|}{\left|T_{k l}\right|} \omega_{k}(1) \omega_{l}(1)\left[\left(\begin{array}{l}
k \\
p
\end{array}\right) \bar{\omega}_{k}(p)+\left(\begin{array}{l}
l \\
p
\end{array}\right) \bar{\omega}_{l}(p)\right] .
$$


By a similar calculation,

$$
\begin{aligned}
\eta\left(p w_{n}\right)= & 2^{p-1}(p-1) ! \frac{\left|C_{W}\left(p w_{n}\right)\right|}{\left|T_{k l}\right|} \omega_{k}(1) \omega_{l}(1) \\
& \cdot\left[\left(\begin{array}{l}
k \\
p
\end{array}\right)(k-p-l) \bar{\omega}_{k}(p)-\left(\begin{array}{l}
l \\
p
\end{array}\right)(l-p-k) \bar{\omega}_{l}(p)\right],
\end{aligned}
$$

where we set $\left(\begin{array}{l}k \\ p\end{array}\right)$ or $\left(\begin{array}{l}l \\ p\end{array}\right)=0$ if $k$ or $l<p$. Setting $\eta(p)=\eta^{\prime}(p)$ and $\eta\left(p w_{n}\right)=\eta^{\prime}\left(p w_{n}\right)$ the lemma follows since

$$
\left|\begin{array}{cc}
1 & 1 \\
k-p-l & -(l-p-k)
\end{array}\right|=2 p \neq 0 .
$$

(In the case where $\left(\begin{array}{l}k \\ p\end{array}\right)$ or $\left(\begin{array}{l}l \\ p\end{array}\right)=0$ the lemma is immediate.)

By Lemma (6.4), in order to complete the proof of Theorem (2.8) for $B_{n}, n \geqq 3$, it is sufficient to prove the following results about characters of the symmetric group $S_{m}$. We shall follow the notations in Murnaghan [14]. Let $\lambda=\left(\lambda_{1}, \ldots, \lambda_{k}\right)$ be a partition of $m$, with $\lambda_{1} \geqq \lambda_{2} \geqq \cdots, \sum \lambda_{i}=m$, and $\omega_{\lambda}$ the character of $S_{m}$ associated with $\lambda$. Let $s$ be the length of the principal diagonal in the corresponding Young tableau, and let $\left(b_{1}, \ldots, b_{s}\right),\left(a_{1}, \ldots, a_{s}\right)$ be defined as in Murnaghan [14, p. 137]. For another character $\omega_{\lambda^{\prime}}$, we let $\lambda_{i}^{\prime}, b_{i}^{\prime}, a_{i}^{\prime}$, etc., be defined in a corresponding way.

(6.7) THEOREM. Let $\lambda, \lambda^{\prime}$ be partitions of $m \geqq 3$, and $\omega_{\lambda}$ and $\omega_{\lambda^{\prime}}$ the corresponding irreducible characters of $S_{m}$. Suppose $\bar{\omega}_{\lambda}(p)=\bar{\omega}_{\lambda^{\prime}}(p)$, for $2 \leqq p \leqq m-1$. Then $\omega_{\lambda}=\omega_{\lambda^{\prime}}$.

Proof. From [14], $\bar{\omega}_{\lambda}(p)$ is the coefficient of $y^{-1}$ in

$$
y(y-1) \cdots(y-p+1) F(y-p) / F(y),
$$

divided by $-p m(m-1) \cdots(m-p+1)$. The function $F(y-p) / F(y)$ has the expansion

$$
1-p\left[F^{\prime}(y) / F(y)\right]+p^{2}\left[F^{\prime \prime}(y) / 2 ! F(y)\right]+\cdots
$$

where $F^{\prime}(y) / F(y)=m / y^{2}+c_{3} / y^{3}+c_{4} / y^{4}+\cdots$ and

$$
c_{i+1}=\sum_{j=1}^{s}\left[b_{j}^{i}+(-1)^{i-1}\left(a_{j}+1\right)^{i}\right], \quad i=2, \ldots
$$

It is easily seen that

$$
F(y-p) / F(y)=A_{0 p}+A_{1 p} / y+A_{2 p} / y^{2}+\cdots
$$

where $A_{i p}=\gamma_{i p} c_{i}+f_{i}\left(m, p, c_{3}, \ldots, c_{i-1}\right), i \geqq 3$, with $\gamma_{i} \neq 0$ and $f_{i}$ independent of $\lambda$. Thus, setting $\bar{\omega}_{\lambda}(p)=\bar{\omega}_{\lambda^{\prime}}(p)$, for $p=2, \ldots, m-1$, we have $c_{i+1}=c_{i+1}^{\prime}, i=2, \ldots$, $m-1$, where $c_{i+1}^{\prime}=\sum_{j=1}^{s}\left[\left(b_{j}^{\prime}\right)^{i}+(-1)^{i-1}\left(a_{j}^{\prime}+1\right)^{i}\right]$. Letting $\bar{a}_{i}=-\left(a_{i}+1\right)$, we have $c_{i+1}$ $=\sum_{j=1}^{s}\left(b_{j}^{i}-\bar{a}_{j}^{i}\right), i=2, \ldots$ Now let

$$
S_{r}(t)=\alpha_{r 1} t+\alpha_{r 2} t^{2}+\cdots
$$


be the polynomial in $t$ such that $S_{r}(u)=1^{r}+2^{r}+\cdots+u^{r}$, for positive integers $u$. Then we have, for $1 \leqq r \leqq m-1, S_{r}(0)=0$, and

$$
\alpha_{r 1} m+\alpha_{r 2} c_{3}+\cdots=\sum_{j=1}^{s}\left[S_{r}\left(b_{j}\right)+(-1)^{r} S_{r}\left(a_{j}\right)\right]
$$

using the identity $S_{r}(-u)=(-1)^{r-1} S_{r}(u-1)$ for positive integers $u$ and the fact that $\operatorname{deg} S_{r}(t)=r+1$. Now, setting $c_{i+1}=c_{i+1}^{\prime}, 2 \leqq i \leqq m-1$, we have for all $r, 1 \leqq r \leqq m-1$,

$$
\sum_{j=1}^{s}\left[S_{r}\left(b_{j}\right)+(-1)^{r} S_{r}\left(a_{j}\right)\right]=\sum_{j=1}^{s^{\prime}}\left[S_{r}\left(b_{j}^{\prime}\right)+(-1)^{r} S_{r}\left(a_{j}^{\prime}\right)\right] \text {. }
$$

Now introduce $m-s$ new variables

$$
\begin{gathered}
u_{1}=1, \quad u_{2}=2, \ldots, \quad u_{b_{1}}=b_{1}, \quad u_{b_{1}+1}=1, \ldots, \\
u_{b_{1}+b_{2}}=b_{2}, \ldots, \quad u_{b_{1}+\cdots+b_{s}}=b_{s}, \\
u_{b_{1}+\cdots+b_{s}+1}=-1, \ldots, \quad u_{b_{1}+b_{2}+\cdots+b_{s}+a_{1}+\cdots+a_{s}}=-a_{s}
\end{gathered}
$$

and similarly introduce $\left\{u_{j}^{\prime}\right\}_{1 \leqq j \leqq m-s}$. Then (6.8) implies that

$$
\sum_{1}^{m-s} u_{i}^{r}=\sum_{1}^{m-s^{\prime}}\left(u_{i}^{\prime}\right)^{r}, \quad 1 \leqq r \leqq m-1 .
$$

Applying Newton's identities we have first, since none of the variables are zero, that $s=s^{\prime}$, and the $u_{i}^{\prime}$ 's and $u_{i}^{\prime \prime}$ s are the same except for a permutation. Now $b_{1}$ $=\max \left\{u_{i}\right\}, b_{1}^{\prime}=\max \left\{u_{i}^{\prime}\right\}$, and the maximums are unique. So $b_{1}=b_{1}^{\prime}$. Similarly $a_{1}=a_{1}^{\prime}$. Cancelling $\left\{1,2, \ldots, b_{1},-1,-2, \ldots,-a_{1}\right\}$ from both sides, we can repeat the argument to obtain $a_{i}=a_{i}^{\prime}, b_{i}=b_{i}^{\prime}, 1 \leqq i \leqq s$. This completes the proof of Theorems (6.7) and (2.8) for the case $B_{n}, n \geqq 3$. The proof of Theorem (2.8) for type $B_{2}$ is left as an exercise.

7. Proof of Theorem (2.9) for $B_{n}$. From Propositions (4.4), (4.5) and (4.7), and the results of $\$ 6$, the proof of Theorem (2.9) for $B_{n}$ is reduced to the proofs of Propositions (7.1) and (7.3) below.

(7.1) Proposition. Let $\eta=\left(\tilde{\psi}_{k l} \omega_{k} \omega_{l}\right)^{W}$ be an irreducible character of the Coxeter group $W$ of type $B_{n}, n>2$, where, as in $\S 6, \psi_{k l} \omega_{k} \omega_{l}$ is an irreducible character of $T_{k l}=B_{k} \times B_{l}$. Then $P=S_{k} \times B_{l}$ is a parabolic subgroup of $W, \chi=\omega_{k}\left(\tilde{\psi}_{0 l} \omega_{l}\right)$ is an irreducible character of $P$, and $\left(\eta, \chi^{W}\right)=1$.

Proof. We have $P=\left\langle w_{1}, \ldots, w_{k-1}, w_{k+1}, \ldots, w_{n}\right\rangle$, and is a parabolic subgroup. We are using the convention that $P=\left\langle w_{1}, \ldots, w_{n}\right\rangle$ or $\left\langle w_{2}, \ldots, w_{n}\right\rangle$ if $k=0$ or 1 respectively, and in these cases $\omega_{k}$ does not appear in $\chi$.

The key to the result is repeated application of Clifford's Theorem [10, Theorem 2]. We first observe that by Frobenius reciprocity every irreducible component of $\chi^{T_{k l}}$ is a component of $\psi_{0 l}^{T}{ }^{k l}$, where $\psi_{0 l}$ is defined on $N_{l}=\langle-(k+1), \ldots,-(n)\rangle$, and sends all the generators of $N_{l}$ to -1 . We have, by an easy computation, $\psi_{l 0}^{N}$ 
$=\sum_{J \subset K} \psi_{J l}$, where $\psi_{J l}$ is the linear character on $N$ which agrees with $\psi_{0 l}$ on $N_{l}$, and for $J \subset K=\{1,2, \ldots, k\}, \psi_{J l}(-(i))=-1$ for $i \in J$ and $\psi_{J l}(-(i))=1$ for $i \notin J$. Then

$$
\psi_{0 l}^{T k l}=\psi_{k l}^{T k l}+\sum_{J \subset K: J \neq \varnothing} \psi_{J l}^{T_{k l}} .
$$

Therefore, by Clifford's Theorem [10], $\chi^{T_{k l}}=\sum_{s \in S} \lambda_{s}+\sum_{t \in T} \mu_{t}$, where the $\left\{\lambda_{s}\right\}_{s \in S}$ are irreducible characters of $T_{k l}$ which are components of $\psi_{k l}^{T k l}$, and the $\left\{\mu_{t}\right\}_{t \in T}$ are irreducible characters of $T_{k l}$ which are components of $\psi_{J l}^{T}$, for $J \neq \varnothing$.

We shall first prove that there is only one character of the form $\lambda_{s}$, and that this character is $\tilde{\psi}_{k l} \omega_{k} \omega_{l}$. An arbitrary character in $\psi_{k l}^{T_{k l}}$ has the form $\tilde{\psi}_{k l} \omega_{k}^{\prime} \omega_{l}^{\prime}$, where $\omega_{k}^{\prime}$ and $\omega_{l}^{\prime}$ are characters of $S_{k}$ and $S_{l}$ respectively. We have $\left(\tilde{\psi}_{k l} \omega_{k}^{\prime} \omega_{l}^{\prime}, \chi^{T_{k l}}\right)_{T_{k l}}$ $=\left(\left.\tilde{\psi}_{k l} \omega_{k}^{\prime} \omega_{l}^{\prime}\right|_{P}, \chi\right)_{P}$, by Frobenius reciprocity. But $\left.\tilde{\psi}_{k i} \omega_{k}^{\prime} \omega_{l}^{\prime}\right|_{P}=\omega_{k}^{\prime}\left(\tilde{\psi}_{0 l} \omega_{l}^{\prime}\right)$, so the above multiplicity is zero unless $\omega_{k}^{\prime}=\omega_{k}, \omega_{l}^{\prime}=\omega_{l}$, and in that case it is one. Thus

$$
\chi^{T_{k l}}=\tilde{\psi}_{k l} \omega_{k} \omega_{l}+\sum_{t \in T} \mu_{t}
$$

We now have, since $\left(\tilde{\psi}_{k l} \omega_{k} \omega_{l}\right)^{W}=\eta, \chi^{W}=\eta+\sum_{t \in T} \mu_{t}^{W}$. But it is easily seen that the irreducible components of the $\mu_{t}^{W}$ are all components of characters $\psi_{k^{\prime} l^{\prime}}^{W}$, with $l^{\prime}>l$, and cannot contribute another $\eta$ to the formula for $\chi^{w}$. This completes the proof.

(7.3) Proposition. Let $P$ and $\chi$ be as in Proposition (7.1). Then there exists a parabolic subgroup $P_{1}$ of $P$ such that $\left(\chi, 1_{P_{1}}^{P}\right)=1$.

Proof. From Proposition (5.3), there exist parabolic subgroups $R_{k}$ and $R_{l}$ of $S_{k}$ and $S_{l}$ respectively, such that $\left(1_{R_{k}}^{S_{k}}, \omega_{k}\right)=\left(1_{R_{l}}^{S_{l}}, \omega_{l}\right)=1$. Using the fact that $\left.\left(\tilde{\psi}_{0 l} \omega_{l}\right)\right|_{R_{l}}$ $=\left.\omega_{l}\right|_{R_{l}}$ and Frobenius reciprocity, we have $\left(1_{R_{l}}^{B_{l}}, \tilde{\psi}_{0 l} \omega_{l}\right)=1$. The conclusion of Proposition (7.3) follows by taking $P_{1}=R_{k} \times R_{l}$.

In applying Proposition (4.5), it is essential to have the fact that the characters of a generic algebra of type $A_{k} \times B_{l}$ are rational, and this follows from Proposition (4.7) and the results in $\$ \S 5$ and 6. This completes the proof of Theorem (2.9) for type $B_{n}, n>2$. The case of $B_{2}$ is easily handled since every character is of parabolic type.

8. Rationality of characters of a generic algebra of type $D_{n}$. Let $(W, R)$ be of type $D_{n}, n \geqq 4$. We shall view $W$ as a subgroup of index two in the Coxeter group $W\left(B_{n}\right)$ of type $B_{n}$; and may take the set $R$ to be $\left\{w_{1}, \ldots, w_{n}\right\}$, with $w_{i}=(i, i+1)$, $1 \leqq i \leqq n-1$, and $w_{n}=(n,-(n-1))$. From Carter [2, Proposition 25], two elements of $W$ are conjugate (in $W$ ) if and only if they have the same signed cycle type, with the exception that there are two classes having the same signed cycle type if all the cycles are even and positive. As in $\$ \S 5$ and 6 , we shall prove that an irreducible character $\zeta$ of $W$ is determined by its multiplicities $\left(\zeta, 1_{W_{J}}^{W}\right)$, for subsets $J \subset R$. We shall make constant use of the corresponding results for $W\left(B_{n}\right)$. 
(8.1) Proposition. Let $\zeta$, $\zeta^{\prime}$ be irreducible characters of $W$ such that $\left(\zeta-\zeta^{\prime}, 1_{w_{J}}^{W}\right)$ $=0$ for all $J \subset R$. Then $\zeta(x)=\zeta^{\prime}(x)$ for all elements $x$ belonging to the parabolic subgroups $\left\langle w_{1}, \ldots, w_{n-1}\right\rangle$, and $\left\langle w_{1}, \ldots, w_{n-3}, w_{n-1}, w_{n}\right\rangle$.

The proof is similar to that of Proposition (5.1) and is omitted.

We shall use the notation $\psi^{*}$ for $\psi^{W\left(B_{n}\right)}$ for characters $\psi$ of subgroups of $W\left(B_{n}\right)$. Then $\zeta^{*} \mid W=\zeta+\zeta^{g}$, for $g \in W\left(B_{n}\right), g \notin W$. It follows that if $\zeta=\zeta^{g}$, then $\zeta^{*}=\eta+\varepsilon \eta$, for some irreducible character $\eta$ of $B_{n}$, and a linear character $\varepsilon \neq 1$ of $W\left(B_{n}\right)$ such that $\varepsilon \mid W=1$. If $\zeta \neq \zeta^{g}$, then $\zeta^{*}$ is irreducible.

We shall first prove Theorem (2.8) for $W$ of type $D_{n}$, with $n \geqq 4$ and $n$ odd. In this case, two elements in $W$ which are conjugate in $W\left(B_{n}\right)$ are conjugate in $W$, since not all cycles can be even in this case. It follows that if $\zeta$ and $\zeta^{\prime}$ are as in Proposition (8.1), then $\zeta=\zeta^{g}$ for all $g \in W\left(B_{n}\right)$, and hence

$$
\zeta^{*}=\eta+\varepsilon \eta, \quad\left(\zeta^{\prime}\right)^{*}=\eta^{\prime}+\varepsilon \eta^{\prime},
$$

for irreducible characters $\eta$ and $\eta^{\prime}$ of $W\left(B_{n}\right)$. Moreover, by Proposition (8.1), $\eta(x)=\eta^{\prime}(x)$ for $x$ in one of the parabolic subgroups of $W$ listed in Proposition (8.1).

(8.2) Proposition. Let the characters $\eta$ and $\eta^{\prime}$ defined above have the forms $\eta=\left(\tilde{\psi}_{k l} \omega_{k} \omega_{l}\right)^{*}, \eta^{\prime}=\left(\tilde{\psi}_{k^{\prime} l^{\prime}} \omega_{k^{\prime}}^{\prime} \omega_{l^{\prime}}^{\prime}\right)^{*}$. Then $k=k^{\prime}$ and $l=l^{\prime}$ or $k=l^{\prime}$ and $l=k^{\prime}$.

Proof. From Proposition (8.1) we have $\eta(1)=\eta^{\prime}(1)$ and $\eta\left(w_{n-1} w_{n}\right)=\eta^{\prime}\left(w_{n-1} w_{n}\right)$. We have

$$
\eta(1)=\left[W\left(B_{n}\right): T_{k l}\right] \omega_{k}(1) \omega_{l}(1)
$$

and

$$
\eta\left(w_{n-1} w_{n}\right)=\frac{\left|C_{W\left(B_{n}\right)}\left(w_{n-1} w_{n}\right)\right| \omega_{k}(1) \omega_{l}(1)}{\left|T_{k l}\right|}\left[\left(\begin{array}{l}
k \\
2
\end{array}\right)+\left(\begin{array}{l}
l \\
2
\end{array}\right)-k l\right] .
$$

From these formulas we obtain

$$
k(k-1)+l(l-1)-2 k l=k^{\prime}\left(k^{\prime}-1\right)+l^{\prime}\left(l^{\prime}-1\right)-2 k^{\prime} l^{\prime},
$$

and the proposition follows.

The linear character $\varepsilon$ has the property that $\varepsilon(-(i))=-1,1 \leqq i \leqq n$. Therefore

$$
\varepsilon \eta=\varepsilon\left(\tilde{\psi}_{k l} \omega_{k} \omega_{l}\right)^{*}=\left[\left(\varepsilon \mid T_{k l}\right)\left(\tilde{\psi}_{k l} \omega_{k} \omega_{l}\right)\right]^{*} .
$$

Since $\left(\varepsilon \mid T_{k l}\right) \tilde{\psi}_{k l}$ is conjugate by $W\left(B_{n}\right)$ to $\tilde{\psi}_{l k}$ we have

$$
\varepsilon \eta=\varepsilon\left(\tilde{\psi}_{k l} \omega_{k} \omega_{l}\right)^{*}=\left(\tilde{\psi}_{l k} \omega_{l} \omega_{k}\right)^{*} .
$$

Therefore, by Proposition (8.2), we may assume that $k=k^{\prime}$ and $l=l^{\prime}$.

(8.4) Proposition. Let $\eta$ and $\eta^{\prime}$ be as in Proposition (8.2), with $k=k^{\prime}, l=l^{\prime}$. Then $\bar{\omega}_{k}(p)=\bar{\omega}_{l}(p), \bar{\omega}_{l}(p)=\bar{\omega}_{l} \cdot(p)$, for cycles $p$ in $S_{k}$ or $S_{l}$ respectively of lengths $1,2, \ldots$, $n-2$. 
Proof. Recall that $\bar{\omega}_{k}(p)$ means $\omega_{k}(p) / \omega_{k}(1)$, etc. From Proposition (8.1) and the remark before Proposition (8.2) we have

$$
\eta(p)=\eta^{\prime}(p), \quad \eta\left(p w_{n-1} w_{n}\right)=\eta^{\prime}\left(p w_{n-1} w_{n}\right) .
$$

By a calculation similar to the one used to derive (6.6) we have

$$
\begin{aligned}
\eta\left(p w_{n-1} w_{n}\right)= & \frac{\left|C_{W\left(B_{n}\right)}\left(p w_{n-1} w_{n}\right)\right| 2^{p-1}(p-1) ! \omega_{k}(1) \omega_{l}(1)}{\left|T_{k l}\right|} \\
& \cdot\left\{\left(\begin{array}{c}
k \\
p
\end{array}\right)\left[\left(\begin{array}{c}
k-p \\
2
\end{array}\right)+\left(\begin{array}{l}
l \\
2
\end{array}\right)-(k-p) l\right] \bar{\omega}_{k}(p)\right. \\
& \left.+\left(\begin{array}{l}
l \\
p
\end{array}\right)\left[\left(\begin{array}{l}
k \\
2
\end{array}\right)+\left(\begin{array}{c}
l-p \\
2
\end{array}\right)-(l-p) k\right] \bar{\omega}_{l}(p)\right\} .
\end{aligned}
$$

The proposition follows from (6.5) and (8.6) since either $k<p$ or $l<p$, or the coefficient determinant for the resulting system of equations for $\bar{\omega}_{k}(p)-\bar{\omega}_{k}^{\prime}(p)$ and $\bar{\omega}_{l}(p)-\bar{\omega}_{l}^{\prime}(p)$ is $c(k-l)$, for some positive constant $c$, and since $n$ is odd, $k-l \neq 0$.

In the proof of Theorem (6.7), the hypothesis actually used was that $\bar{\omega}_{\lambda}(p)$ $=\bar{\omega}_{\lambda^{\prime}}(p)$, for $p=2, \ldots, \max \left\{m-s, m-s^{\prime}\right\}$, where $s$ and $s^{\prime}$ are the lengths of the principal diagonals in the Young tableaus for the partitions $\lambda$ and $\lambda^{\prime}$. Therefore, from Proposition (8.4), we can apply Theorem (6.7) to conclude that $\omega_{k}=\omega_{k}^{\prime}$ and $\omega_{l}=\omega_{l}^{\prime}$, and hence $\zeta=\zeta^{\prime}$, or else $k$ or $l$ is equal to $n$. In the latter case, we have $\eta(p)=\eta^{\prime}(p)$ for all $p$, and hence by Theorem (6.7) again, we obtain $\zeta=\zeta^{\prime}$. This completes the proof of Theorem (2.8) for type $D_{n}$, with $n$ odd.

We assume now that $n$ is even, and as before, that $\zeta$ and $\zeta^{\prime}$ are irreducible characters of $W$ such that $\left(\zeta-\zeta^{\prime}, 1_{W_{J}}^{W}\right)=0$ for all $J \subset R$. Let $g=-(n)$; then $W\left(B_{n}\right)$ $=W \cup g W$. We shall consider three cases.

Case 1. $\zeta^{g}=\zeta,\left(\zeta^{\prime}\right)^{g}=\zeta^{\prime}$. We proceed as in the case of $n$ odd, using Propositions (8.2) and (8.4). In case $k \neq l$, we obtain $\zeta=\zeta^{\prime}$ as before. Now suppose $k=l$. From (6.5) we have (recalling that $\omega_{k}$ and $\omega_{l}$ are not necessarily the same),

$$
\bar{\omega}_{k}(p)+\bar{\omega}_{l}(p)=\bar{\omega}_{k}^{\prime}(p)+\bar{\omega}_{l}^{\prime}(p),
$$

for $p=2, \ldots, n-2$. Now let $p$ and $q$ be disjoint cycles in $\left\langle w_{1}, \ldots, w_{n-3}\right\rangle$ of lengths $p$ and $q$ respectively. Then by Proposition (8.1), we have

$$
\eta(p q)=\eta^{\prime}(p q), \quad \eta\left(p q w_{n-1} w_{n}\right)=\eta^{\prime}\left(p q w_{n-1} w_{n}\right) .
$$

In case $p$ and $q$ have different lengths, we have

$$
\eta(p q)=\left|T_{k l}\right|^{-1} 2^{p+q-2}(p-1) !(q-1) ! \omega_{k}(1) \omega_{l}(1)\left|C_{W\left(B_{n}\right)}(p q)\right| F_{p q}
$$

where

$$
\begin{aligned}
F_{p q}=\left[\left(\begin{array}{l}
k \\
p
\end{array}\right)\left(\begin{array}{l}
l \\
q
\end{array}\right) \bar{\omega}_{k}(p) \bar{\omega}_{l}(q)+\left(\begin{array}{l}
k \\
q
\end{array}\right)\left(\begin{array}{l}
l \\
p
\end{array}\right) \bar{\omega}_{k}(q) \bar{\omega}_{l}(p)\right. \\
\left.+\left(\begin{array}{c}
k \\
p
\end{array}\right)\left(\begin{array}{c}
k-p \\
q
\end{array}\right) \bar{\omega}_{k}(p q)+\left(\begin{array}{l}
l \\
p
\end{array}\right)\left(\begin{array}{c}
l-p \\
q
\end{array}\right) \bar{\omega}_{l}(p q)\right] .
\end{aligned}
$$


If $p$ and $q$ have equal lengths then the formula for $\eta(p q)$ has to be multiplied by $\frac{1}{2}$. Similarly, for $p$ and $q$ of unequal length, we have

$$
\begin{aligned}
& \eta\left(p q w_{n-1} w_{n}\right) \\
& \quad=\left|T_{k l}\right|^{-1} 2^{p+q-2}(p-1) !(q-1) ! \omega_{k}(1) \omega_{l}(1)\left|C_{W\left(B_{n}\right)}\left(p q w_{n-1} w_{n}\right)\right| G_{p q}
\end{aligned}
$$

where

$$
\begin{aligned}
G_{p q}=\left\{( \begin{array} { l } 
{ k } \\
{ p }
\end{array} ) ( \begin{array} { l } 
{ l } \\
{ q }
\end{array} ) \left[\left(\begin{array}{c}
k-p \\
2
\end{array}\right)\right.\right. & \left.+\left(\begin{array}{c}
l-q \\
2
\end{array}\right)-(k-p)(l-q)\right] \bar{\omega}_{k}(p) \bar{\omega}_{l}(q) \\
& +\left(\begin{array}{l}
k \\
q
\end{array}\right)\left(\begin{array}{l}
l \\
p
\end{array}\right)\left[\left(\begin{array}{c}
k-q \\
2
\end{array}\right)+\left(\begin{array}{c}
l-p \\
2
\end{array}\right)+(k-q)(l-p)\right] \bar{\omega}_{k}(q) \bar{\omega}_{l}(p) \\
& +\left(\begin{array}{c}
k \\
p
\end{array}\right)\left(\begin{array}{c}
k-p \\
q
\end{array}\right)\left[\left(\begin{array}{c}
k-p-q \\
2
\end{array}\right)+\left(\begin{array}{l}
l \\
2
\end{array}\right)-(k-p-q) l\right] \bar{\omega}_{k}(p q) \\
& \left.+\left(\begin{array}{l}
l \\
p
\end{array}\right)\left(\begin{array}{c}
l-p \\
q
\end{array}\right)\left[\left(\begin{array}{c}
l-p-q \\
2
\end{array}\right)+\left(\begin{array}{l}
k \\
2
\end{array}\right)-(l-p-q) k\right] \bar{\omega}_{l}(p q)\right\} .
\end{aligned}
$$

Again, if $p$ and $q$ have the same length, the expression is multiplied by $\frac{1}{2}$.

Since $k=l,(8.8),(8.9)$ and (8.10) give a system of homogeneous equations in

$$
A_{p q}=\left(\bar{\omega}_{k}(p) \bar{\omega}_{l}(q)+\bar{\omega}_{k}(q) \bar{\omega}_{l}(p)\right)-\left(\bar{\omega}_{k}^{\prime}(p) \bar{\omega}_{l}^{\prime}(q)+\bar{\omega}_{k}^{\prime}(q) \bar{\omega}_{l}^{\prime}(p)\right)
$$

and

$$
B_{p q}=\left(\bar{\omega}_{k}(p q)+\bar{\omega}_{l}(p q)\right)-\left(\bar{\omega}_{k}^{\prime}(p q)+\bar{\omega}_{l}^{\prime}(p q)\right) .
$$

The coefficient determinant is a nonzero multiple of $p q$, and we obtain that $A_{p q}=0$ in (8.11) for all disjoint cycles $p$ and $q$ in $\left\langle w_{1}, \ldots, w_{n-3}\right\rangle$. In particular, if $p$ and $q$ have the same length, then, viewing $\omega_{k}, \omega_{k}^{\prime}, \omega_{l}, \omega_{l}^{\prime}$ as characters of the symmetric group $S_{k}$, and $p$ as a $p$-cycle in $S_{k}$, we have, for $p=2, \ldots, k-1$,

$$
\bar{\omega}_{k}(p) \bar{\omega}_{l}(p)=\bar{\omega}_{k}^{\prime}(p) \bar{\omega}_{l}^{\prime}(p) .
$$

Combining (8.12) with (8.7) we have

$$
\left\{\bar{\omega}_{k}(p), \bar{\omega}_{l}(p)\right\}=\left\{\bar{\omega}_{k}^{\prime}(p), \bar{\omega}_{l}^{\prime}(p)\right\},
$$

for $2 \leqq p \leqq k-1$. If either $\bar{\omega}_{k}(p)=\bar{\omega}_{k}^{\prime}(p), 2 \leqq p \leqq k-1$, or $\bar{\omega}_{k}(p)=\bar{\omega}_{l}^{\prime}(p), 2 \leqq p \leqq k-1$, we have, by Theorem (6.7) and (8.13), that $\omega_{k}=\omega_{k}^{\prime}$ and $\omega_{l}=\omega_{l}^{\prime}$ or $\omega_{k}=\omega_{l}^{\prime}$ and $\omega_{l}=\omega_{k}^{\prime}$, respectively. Otherwise there exist cycles $p$ and $q$, of lengths less than $k$, such that

$$
\begin{array}{ll}
\bar{\omega}_{k}(p)=\bar{\omega}_{l}^{\prime}(p), & \bar{\omega}_{k}(p) \neq \bar{\omega}_{k}^{\prime}(p) \\
\bar{\omega}_{k}(q)=\bar{\omega}_{k}^{\prime}(q), & \bar{\omega}_{k}(q) \neq \bar{\omega}_{l}^{\prime}(q) .
\end{array}
$$

From (8.13) and (8.14), we have

$$
\bar{\omega}_{l}(p)=\bar{\omega}_{k}^{\prime}(p), \quad \bar{\omega}_{l}(q)=\bar{\omega}_{l}^{\prime}(q) .
$$


Using the fact that $A_{p q}=0$ in (8.11), together with (8.14) and (8.15), yields

$$
\bar{\omega}_{k}(p) \bar{\omega}_{l}(q)+\bar{\omega}_{k}(q) \bar{\omega}_{l}(p)=\bar{\omega}_{l}(p) \bar{\omega}_{l}(q)+\bar{\omega}_{k}(q) \bar{\omega}_{k}(p),
$$

from which it follows that

$$
\left[\bar{\omega}_{k}(p)-\bar{\omega}_{l}(p)\right]\left[\bar{\omega}_{l}(q)-\bar{\omega}_{k}(q)\right]=0 .
$$

If $\bar{\omega}_{k}(p)=\bar{\omega}_{l}(p)$ then from (8.15) we have $\bar{\omega}_{k}(p)=\bar{\omega}_{k}^{\prime}(p)$ contradicting (8.14). Similarly, if $\bar{\omega}_{l}(q)=\bar{\omega}_{k}(q)$ we obtain from $(8.15)$ that $\bar{\omega}_{k}(q)=\bar{\omega}_{l}^{\prime}(q)$, contradicting (8.14) again. This completes the discussion of Case 1.

Case 2. $\zeta \neq \zeta^{g}, \zeta^{\prime} \neq\left(\zeta^{\prime}\right)^{g}$. In this case $\eta=\zeta^{*}$ and $\eta^{\prime}=\left(\zeta^{\prime}\right)^{*}$ are both irreducible, and $\eta\left|W=\zeta+\zeta^{g}, \eta^{\prime}\right| W=\zeta^{\prime}+\left(\zeta^{\prime}\right)^{g}$.

(8.16) Proposition. Suppose $\left(\zeta-\zeta^{\prime}, 1_{P}^{W}\right)=0$ for all parabolic subgroups $P$ of $W$. Then $\left(\zeta^{g}-\left(\zeta^{\prime}\right)^{g}, 1_{P}^{W}\right)=0$ for all such $P$.

Proof. It is sufficient to prove that if $P$ is a parabolic subgroup of $W$, then so is $P^{g}$, for $g=-(n)$. The proposition follows since $w_{i}^{g}=w_{i}, 1 \leqq i \leqq n-2$, and $w_{n-1}^{g}=w_{n}$ $w_{n}^{g}=w_{n-1}$.

Proposition (8.16) implies that $\left(\eta-\eta^{\prime}, 1_{P}^{*}\right)=0$ for all parabolic subgroups $P$ of $W$. Using Proposition (8.2) and the discussion in Case 1, we obtain either $\eta=\eta^{\prime}$ or, by (8.3), $\eta=\varepsilon \eta^{\prime}$ where $\varepsilon \mid W=1$. Restricting to $W$ gives $\zeta=\zeta^{\prime}$ or $\zeta^{g}=\zeta^{\prime}$. Suppose $\zeta^{g}=\zeta^{\prime}$. Then $\left(\zeta^{g}-\zeta, 1_{P}^{W}\right)=0$ for all parabolic subgroups $P$ of $W$.

(8.17) Proposition. If $\left(\zeta^{g}-\zeta, 1_{P}^{W}\right)=0$ for all parabolic subgroups $P$ of $W$, then $\zeta=\zeta^{g}$.

Proof. If $\zeta(x) \neq \zeta^{g}(x)$, then all cycles of $x$ are even and positive, by Carter [2, Proposition 25]. There exists an element $y$ of the same cycle type as $x$ in $W_{1}$ $=\left\langle w_{1}, \ldots, w_{n-1}\right\rangle$. By Carter [2, Proposition 25], $x$ is conjugate in $W$ either to $y$ or $y^{g}$, since $W\left(B_{n}\right)=W \cup g W$. Now, $W_{1}$ and $W_{1}^{g}=\left\langle w_{1}, \ldots, w_{n-2}, w_{n}\right\rangle$ are both parabolic in $W$ and are of type $A_{n-1}$. By an argument similar to Proposition (5.1), we conclude that if $\zeta(y) \neq \zeta^{g}(y)$, for $y \in W_{1}$, there exists a parabolic subgroup $P_{1}$ of $W_{1}$ such that $\left(\zeta-\zeta^{g}, 1_{P_{1}}^{W}\right) \neq 0$. This completes the proof of Proposition (8.17), and finishes the discussion of Case 2, since it now follows that $\zeta=\zeta^{\prime}$.

Case 3. $\zeta \neq \zeta^{g}, \zeta^{\prime}=\left(\zeta^{\prime}\right)^{g}$. In this case, Proposition (8.16) implies that $\left(\zeta^{g}-\zeta^{\prime}, 1_{P}^{W}\right)$ $=0$ for all parabolic subgroups $P \subset W$. Thus $\left(\zeta^{g}-\zeta, 1_{P}^{W}\right)=0$ for all such $P$, and $\zeta=\zeta^{g}$ by Proposition (8.17), contradicting the hypothesis.

This completes the proof of Theorem (2.8) for type $D_{n}, n \geqq 4$.

9. Proof of Theorem (2.9) for $D_{n}$. The proof of Theorem (2.9) for $W=D_{n}$, $n \geqq 4$, follows from Propositions (7.1), (9.1), and (9.6), together with the results in $\S \S 4$ and 8.

(9.1) Proposition. Let $\zeta$ be an irreducible character of $W$ of type $D_{n}, n \geqq 4$. Then there exists a parabolic subgroup $P$ of $W$ and an irreducible character $\mu$ of $P$ such that $\left(\zeta, \mu^{W}\right)=1$. 
Proof. Let $\eta=\left(\tilde{\psi}_{k l} \omega_{k} \omega_{l}\right)^{*}$, be an irreducible component of $\zeta^{*}$. From Proposition (7.1), $\chi=\omega_{k}\left(\tilde{\psi}_{0 l} \omega_{l}\right)$ is an irreducible character of $S_{k} \times B_{l}$ and $\left(\eta, \chi^{*}\right)=1$. Let $P$ $=S_{k} \times D_{l}$. Then $P$ is a parabolic subgroup of $W$ and $P^{g}=P$ for $g=-(n)$. Let $\lambda$ be an irreducible component of $\left.\chi\right|_{P}$. We consider four cases:

Case 1. $\zeta^{g} \neq \zeta, \lambda^{g} \neq \lambda$. Then $\eta=\zeta^{*}$ and

$$
\lambda^{w}=a \zeta+b \zeta^{g}+\xi
$$

where $a$ and $b$ are nonnegative integers and neither $\zeta$ nor $\zeta^{g}$ is an irreducible component of $\xi$. Thus $\left(\lambda^{*}, \eta\right)=(a+b)$. Also $\left(\lambda^{*}, \eta\right)=\left(\chi^{*}, \eta\right)=1$ since $\lambda^{S_{k} \times B_{l}}=\chi$. If $a=1, b=0$ we set $\mu=\lambda$. If $a=0, b=1$ we set $\mu=\lambda^{g}$. Then $\left(\mu^{w}, \zeta\right)=1$.

Case 2. $\zeta^{g} \neq \zeta, \lambda^{g}=\lambda$. We have (9.2) and

$$
\lambda^{S_{k} \times B_{l}}=\chi+\varepsilon \chi
$$

where $\varepsilon \neq 1$ is the linear character of $W\left(B_{n}\right)$ which is trivial on $W$. Since $\eta=\zeta^{*}$ and $\varepsilon \mid W=1$, we have $\varepsilon \eta=\eta$ and thus $\left(\lambda^{B_{n}}, \eta\right)=2$. Since $\left(\lambda^{w}\right)^{g}=\left(\lambda^{g}\right)^{W}=\lambda^{W}$, we have from (9.2) that $a=b$. Therefore, the proposition follows in this case with $\mu=\lambda$.

Case 3. $\zeta=\zeta^{g}, \lambda \neq \lambda^{g}$. In this case $\zeta^{*}=\eta+\varepsilon \eta$ and

$$
\lambda^{w}=a \zeta+\xi^{\prime}
$$

where $a$ is a nonnegative integer and $\zeta$ is not an irreducible component of $\xi^{\prime}$. Then $\left(\lambda^{*}, \eta\right)=a$. But $\lambda^{S_{k} \times B_{l}}=\chi$ and hence $\left(\lambda^{*}, \eta\right)=\left(\chi^{*}, \eta\right)=1$. Again the proposition follows with $\mu=\lambda$.

Case 4. $\zeta=\zeta^{g}, \lambda=\lambda^{g}$. Both (9.3) and (9.4) hold in this case and $\left(\lambda^{*}, \eta\right)=\left(\lambda^{*}, \varepsilon \eta\right)$ $=a$. From (9.3) and (7.2) we have

$$
\lambda^{T} k l=\tilde{\psi}_{k l} \omega_{k} \omega_{l}+\varepsilon \tilde{\psi}_{k l} \omega_{k} \omega_{l}+\sum_{t \in T} \theta_{t}+\varepsilon\left(\sum_{t \in T} \theta_{t}\right)
$$

where $\eta=\left(\tilde{\psi}_{k l} \omega_{k} \omega_{l}\right)^{*}$, and $\left\{\theta_{t}\right\}_{t \in T}$ are irreducible characters of $T_{k l}$ which are irreducible components of $\psi_{J, l}^{T_{k l}}$, for $J \neq \varnothing$. From the discussion in $\S 7$, and (8.3), $\left(\sum_{t \in T} \theta_{t}\right)^{*}$ can contain $\varepsilon\left(\tilde{\psi}_{k l} \omega_{k} \omega_{l}\right)^{*}=\left(\tilde{\psi}_{l k} \omega_{l} \omega_{k}\right) *$ only if $k>l$. We may assume, however, that in choosing $\eta, k \leqq l$. Thus $\left(\lambda^{*}, \varepsilon \eta\right)=1$ and thus $\left(\zeta, \mu^{w}\right)=1$ for $\mu=\lambda$.

(9.6) Proposition. Let $\mu$ and $P$ be as in the proof of Proposition (9.1). Then there exists a parabolic subgroup $P_{1}$ of $P$ such that $\left(\mu, 1_{P_{1}}^{P}\right)=1$.

Proof. Let $R_{k}, R_{l}$, and $\chi$ be as in the proof of Proposition (7.3). Then we have $\left(\chi, 1_{R_{k} \times R_{l}}^{S_{k} \times B_{l}}\right)=1$. If $\mu^{S_{k} \times B_{l}}=\chi$, then $\left(\left.\chi\right|_{R_{k} \times R_{l}}, 1_{R_{k} \times R_{l}}\right)=\left(\left.\left(\mu+\mu^{g}\right)\right|_{R_{k} \times R_{l}}, 1_{R_{k} \times R_{l}}\right)=1$. Now both $R_{k} \times R_{l}$ and $\left(R_{k} \times R_{l}\right)^{g}$ are parabolic subgroups of $W$, and by setting $P_{1}$ equal to one of these groups we may ensure $\left(\mu, 1_{P_{1}}^{P}\right)=1$.

If $\mu^{S_{k} \times B_{l}}=\chi+\varepsilon \chi$, we have $\left(\varepsilon \chi, 1_{R_{k} \times R_{l}}^{S_{k} \times B_{l}}\right)=\left(\chi, 1_{R_{k} \times R_{l}}^{S_{k} \times B_{l}}\right)=1$. Therefore $\left(\mu S_{k} \times B_{l}, 1_{R_{k} \times R_{l}}^{S_{k} \times B_{l}}\right)$ $=2$ and since $\left.\mu^{S_{k} \times B_{l}}\right|_{P}=2 \mu$, we have $\left(\left.2 \mu\right|_{R_{k} \times R_{l}}, 1_{R_{k} \times R_{l}}\right)=2$. Thus, setting $P_{1}=R_{k} \times R_{l}$ we have $\left(\left.\mu\right|_{P_{1}}, 1_{P_{1}}\right)=\left(\mu, 1_{P_{1}}^{P}\right)=1$. This completes the proof of Theorem (2.9) for $W$ of type $D_{n}$.

10. Proofs of Theorems (2.8) and (2.9) for the exceptional groups. If $(W, R)$ is of type $G_{2}, F_{4}, E_{6}, E_{7}$, or $E_{8}$, it can be verified directly that the irreducible characters, 
$\zeta$, are determined by the multiplicities, $\left(\zeta, 1_{P}^{W}\right), P$ parabolic, with the exception of the two characters of degree 2 in the case of $G_{2}$, and the two characters of degree 512 in the case of $E_{7}$. If $(W, R)$ is of type $G_{2}$ with $R=\{r, s\}$, it is shown in [4] that the representation of the generic ring corresponding to the reflection representation $W$ is in $Q\left(u^{\left(c_{r}+c_{s}\right) / 2}\right)$. Since we have assumed that $c_{r}+c_{s}$ is even, this representation is rational, and hence so is the representation corresponding to the other irreducible character of $W$ of degree 2. Thus Theorem (2.8) is established.

To prove Theorem (2.9) for the exceptional groups, it is sufficient, by the remarks at the end of $\S 4$, to show that for each irreducible character $\zeta$ of $W, \zeta$ is either of parabolic type or there exists a parabolic subgroup $P$ such that $\left(\zeta, \varepsilon_{P}^{W}\right)=1$, where $\varepsilon_{P}$ is the alternating character $\varepsilon$ of $W$, restricted to $P$. Since for each irreducible character $\zeta$ of $W$, we have $\left(\varepsilon \zeta, 1_{P}^{W}\right)=\left(\zeta, \varepsilon_{P}^{W}\right)$, it is sufficient to show that either $\zeta$ or $\varepsilon \zeta$ is of parabolic type.

In the following tables we list one of the irreducible characters $\zeta$, or $\varepsilon \zeta$, of $W$ followed by the Coxeter element $w_{P}$ of a parabolic subgroup $P$ such that $\left(\zeta, 1_{P}^{W}\right)$ or $\left(\varepsilon \zeta, 1_{P}^{W}\right)=1$. We omit the case of $G_{2}$ where it may be verified that every irreducible character is of parabolic type. In the case of $F_{4}$ all irreducible characters are listed. In labelling the irreducible characters we follow the notation in [12], [8], and [9], except that we write $\zeta^{\prime}$ for $\varepsilon \zeta$ and in the case of $F_{4}$ we write $\chi_{d, i}$ for the $i$ th character of degree $d$ given in the character table in [12]. The generators in $R$ are labeled as in (10.1):

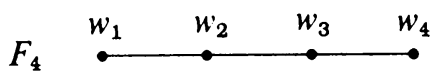

(In [12], $\left.w_{1} \rightarrow d, w_{2} \rightarrow a, w_{3} \rightarrow \tau, w_{4} \rightarrow \tau \sigma.\right)$

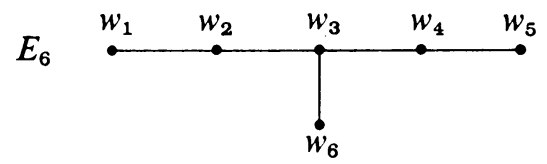

(10.2) $\mathrm{F}_{4}$ :
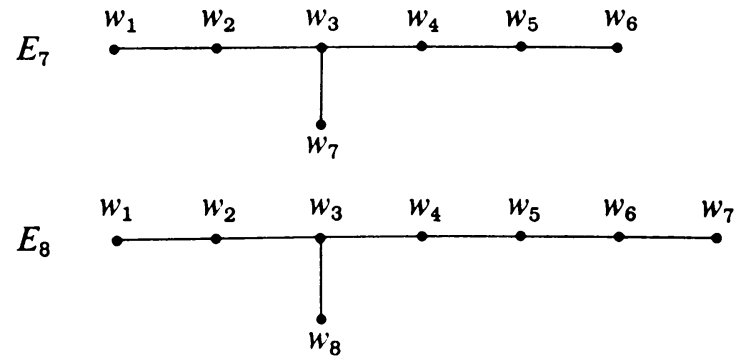

$\begin{array}{llll}\zeta & w_{P} & \zeta & w_{P} \\ \chi_{11} & w_{1} w_{2} w_{3} w_{4} & \chi_{61} & w_{1} w_{2} \\ \chi_{12} & w_{1} w_{2} w_{3} w_{4} & \chi_{62} & w_{2} w_{3} \\ \chi_{13} & w_{1} w_{2} w_{3} w_{4} & \chi_{12,1} & w_{2} w_{3} \\ \chi_{14} & w_{1} w_{2} w_{3} w_{4} & \chi_{42} & w_{1} w_{2} w_{3}\end{array}$




$\begin{array}{llll}\chi_{21} & w_{3} & \chi_{43} & w_{3} \\ \chi_{22} & w_{3} & \chi_{44} & w_{1} \\ \chi_{23} & w_{1} & \chi_{45} & w_{1} \\ \chi_{24} & w_{1} & \chi_{81} & w_{3} w_{4} \\ \chi_{41} & w_{2} w_{3} & \chi_{82} & w_{3} w_{4} \\ \chi_{91} & w_{1} w_{2} w_{3} & \chi_{83} & w_{1} w_{2} \\ \chi_{92} & w_{2} w_{3} & \chi_{84} & w_{1} w_{3} \\ \chi_{93} & w_{2} w_{3} & \chi_{16,1} & w_{1} w_{3} w_{4} \\ \chi_{94} & w_{1} w_{3} & & \end{array}$

(10.3) $E_{6}$ :

$\begin{array}{llll}\zeta & w_{P} & \zeta & w_{P} \\ 1_{p} & w_{1} w_{2} w_{3} w_{4} w_{5} w_{6} & 24_{p}^{\prime} & w_{1} w_{3} w_{5} \\ 6_{p}^{\prime} & w_{1} & 60_{p} & w_{1} w_{2} w_{3} w_{4} \\ 15_{p} & w_{2} w_{3} w_{4} w_{6} & 20_{s} & w_{1} w_{3} w_{5} \\ 20_{p} & w_{1} w_{2} w_{3} w_{4} w_{5} & 90_{s} & w_{1} w_{2} w_{3} w_{5} \\ 30_{p}^{\prime} & w_{1} w_{3} w_{5} & 80_{s} & w_{1} w_{2} w_{3} w_{5} \\ 64_{p}^{\prime} & w_{1} w_{2} w_{4} & 60_{s} & w_{1} w_{2} w_{3} \\ 81_{p} & w_{1} w_{2} w_{3} w_{4} & 10_{s} & w_{1} w_{2} \\ 15_{q}^{\prime} & w_{1} w_{3} w_{5} & & \end{array}$

(10.4) $E_{7}$ :

$\begin{array}{llll}\zeta & w_{P} & \zeta & w_{P} \\ 1 & w_{1} w_{2} w_{3} w_{4} w_{5} w_{6} w_{7} & 405_{a} & w_{1} w_{2} w_{3} w_{4} w_{5} \\ 7_{a} & w_{1} & 168_{a}^{\prime} & w_{1} w_{2} w_{4} w_{6} \\ 27_{a}^{\prime} & w_{1} w_{3} & 56_{a} & w_{1} w_{3} w_{5} \\ 21_{a}^{\prime} & w_{1} w_{3} & 120_{a}^{\prime} & w_{1} w_{2} w_{4} \\ 35_{a} & w_{1} w_{3} w_{5} & 210_{a}^{\prime} & w_{1} w_{2} w_{4} w_{6} \\ 105_{a} & w_{1} w_{2} w_{4} & 280_{a} & w_{1} w_{2} w_{4} w_{5} \\ 189_{a}^{\prime} & w_{1} w_{2} w_{4} w_{5} & 336_{a} & w_{1} w_{2} w_{3} w_{4} \\ 21_{b} & w_{1} w_{3} & 216_{a} & w_{1} w_{2} w_{3} w_{5} \\ 35_{b}^{\prime} & w_{1} w_{3} w_{5} & 512_{a} & w_{1} w_{2} w_{3} w_{4} w_{6} \\ 189_{b} & w_{1} w_{2} w_{4} w_{6} & 378_{a} & w_{2} w_{4} w_{5} w_{6} w_{7} \\ 189_{c} & w_{1} w_{2} w_{4} w_{6} & 84_{a}^{\prime} & w_{1} w_{2} w_{3} \\ 15_{a} & w_{1} w_{3} w_{5} & 420_{a}^{\prime} & w_{1} w_{2} w_{3} w_{4} \\ 105_{b}^{\prime} & w_{1} w_{2} w_{4} w_{6} & 280_{b} & w_{1} w_{2} w_{3} w_{4} w_{5} \\ 105_{c}^{\prime} & w_{1} w_{2} w_{4} w_{5} & 210_{b} & w_{1} w_{2} w_{3} w_{4} \\ 315_{a}^{\prime} & w_{1} w_{2} w_{3} w_{4} w_{5} & 70_{a} & w_{1} w_{2} w_{4} w_{5}\end{array}$

(10.5) $E_{8}$ :

$\begin{array}{llll}\zeta & w_{P} & \zeta & w_{P} \\ 1_{x} & w_{1} w_{2} w_{3} w_{4} w_{5} w_{6} w_{7} w_{8} & 4200_{y} & w_{1} w_{2} w_{3} w_{4} w_{5} w_{7} \\ 28_{x}^{\prime} & w_{1} w_{3} & 2688_{y} & w_{1} w_{2} w_{3} w_{4} w_{5} w_{7}\end{array}$




\begin{tabular}{|c|c|c|c|}
\hline $35_{x}^{\prime}$ & $w_{1} w_{3}$ & $2100_{y}$ & $w_{1} w_{2} w_{3} w_{4} w_{5}$ \\
\hline $84_{x}^{\prime}$ & $w_{1} w_{3} w_{5}$ & $1400_{y}$ & $w_{1} w_{2} w_{3} w_{4} w_{5}$ \\
\hline $50_{x}^{\prime}$ & $w_{1} w_{3} w_{5} w_{7}$ & $4536_{y}$ & $w_{1} w_{2} w_{3} w_{4} w_{5} w_{7}$ \\
\hline $350_{x}^{\prime}$ & $w_{1} w_{2} w_{4} w_{5}$ & $5670_{y}$ & $w_{1} w_{2} w_{3} w_{4} w_{5} w_{7}$ \\
\hline $300_{x}^{\prime}$ & $w_{1} w_{2} w_{4} w_{5}$ & $4480_{y}$ & $w_{1} w_{2} w_{3} w_{4} w_{5} w_{7}$ \\
\hline $567_{x}^{\prime}$ & $w_{1} w_{2} w_{3}$ & $8_{z}^{\prime}$ & $w_{1}$ \\
\hline $210_{x}^{\prime}$ & $w_{1} w_{2} w_{4}$ & $56_{z}$ & $w_{1} w_{2} w_{3} w_{4} w_{5}$ \\
\hline $840_{x}$ & $w_{1} w_{2} w_{3} w_{4} w_{5}$ & $160_{z}^{\prime}$ & $w_{1} w_{2} w_{4}$ \\
\hline $700_{x}^{\prime}$ & $w_{1} w_{2} w_{4} w_{5}$ & $112_{z}^{\prime}$ & $w_{1} w_{2}$ \\
\hline $175_{x}^{\prime}$ & $w_{1} w_{2} w_{4} w_{5}$ & $840_{z}^{\prime}$ & $w_{1} w_{2} w_{3} w_{5} w_{7}$ \\
\hline $1400_{x}^{\prime}$ & $w_{1} w_{2} w_{3} w_{5} w_{7}$ & $1296_{z}^{\prime}$ & $w_{1} w_{2} w_{3} w_{4}$ \\
\hline $1050_{x}^{\prime}$ & $w_{1} w_{2} w_{3} w_{5} w_{7}$ & $1400_{z}^{\prime}$ & $w_{1} w_{2} w_{3} w_{5}$ \\
\hline $1575_{x}^{\prime}$ & $w_{1} w_{2} w_{3} w_{5} w_{7}$ & $1008_{z}^{\prime}$ & $w_{1} w_{2} w_{3} w_{5}$ \\
\hline $1344_{x}^{\prime}$ & $w_{1} w_{2} w_{3} w_{5}$ & $560_{z}^{\prime}$ & $w_{1} w_{2} w_{4} w_{6}$ \\
\hline $2100_{x}^{\prime}$ & $w_{1} w_{2} w_{3} w_{4} w_{6}$ & $1400_{z z}^{\prime}$ & $w_{1} w_{2} w_{3} w_{5} w_{6}$ \\
\hline $2268_{x}^{\prime}$ & $w_{1} w_{2} w_{3} w_{4}$ & $4200_{z}$ & $w_{1} w_{2} w_{3} w_{4} w_{5} w_{6}$ \\
\hline $525_{x}^{\prime}$ & $w_{1} w_{2} w_{3} w_{5}$ & $400_{z}^{\prime}$ & $w_{1} w_{2} w_{4} w_{6} w_{8}$ \\
\hline $700_{x x}$ & $w_{1} w_{2} w_{3} w_{4} w_{5} w_{7}$ & $3240_{z}^{\prime}$ & $w_{1} w_{2} w_{3} w_{5} w_{6}$ \\
\hline $972_{x}^{\prime}$ & $w_{1} w_{2} w_{3} w_{5} w_{7}$ & $4536_{z}^{\prime}$ & $w_{1} w_{4} w_{5} w_{6} w_{7} w_{8}$ \\
\hline $4096_{x}^{\prime}$ & $w_{1} w_{2} w_{3} w_{4} w_{6}$ & $2400_{z}^{\prime}$ & $w_{1} w_{4} w_{5} w_{6} w_{7} w_{8}$ \\
\hline $4200_{x}^{\prime}$ & $w_{1} w_{4} w_{5} w_{6} w_{7} w_{8}$ & $3360_{z}^{\prime}$ & $w_{1} w_{4} w_{5} w_{6} w_{7} w_{8}$ \\
\hline $2240_{x}^{\prime}$ & $w_{1} w_{2} w_{5} w_{6} w_{7} w_{8}$ & $2800_{z}^{\prime}$ & $w_{1} w_{2} w_{3} w_{4} w_{6}$ \\
\hline $2835_{x}$ & $w_{1} w_{2} w_{3} w_{4} w_{5} w_{6}$ & $4096_{z}^{\prime}$ & $w_{1} w_{2} w_{3} w_{4} w_{6}$ \\
\hline $6075_{x}^{\prime}$ & $w_{1} w_{2} w_{3} w_{5} w_{6} w_{7}$ & $5600_{z}^{\prime}$ & $w_{1} w_{2} w_{3} w_{4} w_{5}$ \\
\hline $3200_{x}^{\prime}$ & $w_{1} w_{2} w_{3} w_{4} w_{5}$ & $448_{z}^{\prime}$ & $w_{1} w_{2} w_{4} w_{5} w_{7}$ \\
\hline $70_{y}$ & $w_{1} w_{2} w_{4} w_{5}$ & $448_{w}$ & $w_{1} w_{2} w_{3} w_{4} w_{6}$ \\
\hline $1134_{y}$ & $w_{1} w_{4} w_{5} w_{6} w_{7} w_{8}$ & $1344_{w}$ & $w_{1} w_{2} w_{3} w_{5} w_{6} w_{7} w_{8}$ \\
\hline $1680_{y}$ & $w_{1} w_{2} w_{3} w_{4} w_{5}$ & $5600_{w}$ & $w_{1} w_{2} w_{3} w_{4} w_{5} w_{7}$ \\
\hline $168_{y}$ & $w_{1} w_{2} w_{5} w_{6} w_{7} w_{8}$ & $2016_{w}$ & $w_{1} w_{2} w_{3} w_{4} w_{5}$ \\
\hline $420_{y}$ & $w_{1} w_{2} w_{3} w_{4}$ & $7168_{w}$ & $w_{1} w_{2} w_{3} w_{5} w_{6} w_{7} w_{8}$ \\
\hline $3150_{y}$ & $w_{1} w_{2} w_{3} w_{4} w_{5}$ & & \\
\hline
\end{tabular}

\section{REFERENCES}

1. M. Benard, On the Schur indices of the characters of the exceptional Weyl groups, Ph.D. Dissertation, Yale University, New Haven, Conn., 1969.

2. R. W. Carter, Conjugacy classes in the Weyl group (to appear).

3. C. W. Curtis and T. V. Fossum, On centralizer rings and characters of representations of finite groups, Math. Z. 107 (1968), 402-406. MR 38 \#5946.

4. C. W. Curtis, N. Iwahori and R. Kilmoyer, Hecke algebras and characters of parabolic type of finite groups with $(B, N)$-pairs (to appear).

5. C. W. Curtis and I. Reiner, Representation theory of finite groups and associative algebras, Pure and Appl. Math., vol. 11, Interscience, New York, 1962. MR 26 \#2519.

6. T. V. Fossum, Characters and centers of symmetric algebras, J. Algebra 16 (1970), 4-13. MR 41 \#6894. 
7. R. F. Fox, A simple new method for calculating the characters of symmetric groups, J. Combinatorial Theory 2 (1967), 186-212. MR 34 \#7678.

8. J. S. Frame, The classes and representations of the groups of 27 lines and 28 bitangents, Ann. Mat. Pura Appl. (4) 32 (1951), 83-119. MR 13, 817.

9. - The characters of the Weyl group $E_{8}$, Computational Problems in Abstract Algebra (Proc. Conf. Oxford, 1967), Pergamon, Oxford, 1970, pp. 111-130.

10. P. X. Gallagher, Group characters and normal Hall subgroups, Nagoya Math. J. 21 (1962), 223-230. MR 26 \#240.

11. G. J. Janusz, Primitive idempotents in group algebras, Proc. Amer. Math. Soc. 17 (1966), 520-523. MR 33 \#2733.

12. T. Kondo, The characters of the Weyl group of type $F_{4}$, J. Fac. Sci. Univ. Tokyo Sect. I 11 (1965), 145-153. MR 32 \#2488.

13. S. Lang, Diophantine geometry, Interscience Tracts in Pure and Appl. Math., no. 11, Interscience, New York, 1962. MR 26 \#119.

14. F. D. Murnaghan, The theory of group representations, Johns Hopkins Press, Baltimore, Maryland, 1938.

15. T. Nakayama, Some remarks on regular representations, induced representations, and modular representations, Ann. of Math. 39 (1938), 361-369.

16. T. A. Springer, Cusp forms for finite groups, Lecture Notes in Math., no. 131, SpringerVerlag, New York and Berlin, 1970, (C-1)-(C-24).

17. R. Steinberg, A geometric approach to the representations of the full linear group over a Galois field, Trans. Amer. Math. Soc. 71 (1951), 274-282. MR 13, 317.

18. - Lectures on Chevalley groups, Lecture Notes, Yale University, New Haven, Conn., 1967.

Department of Mathematics, University of Oregon, Eugene, Oregon 97403 